KCL-MTH-06-10

hep-th/0608184

\title{
The logarithmic triplet theory with boundary
}

\author{
Matthias R. Gaberdiel ${ }^{a *}$ and Ingo Runkel ${ }^{b \dagger}$ \\ ${ }^{a}$ Institute for Theoretical Physics, ETH Zürich \\ 8093 Zürich, Switzerland \\ ${ }^{b}$ Department of Mathematics, King's College London \\ Strand, London WC2R 2LS, United Kingdom
}

August 2006

\begin{abstract}
The boundary theory for the $c=-2$ triplet model is investigated in detail. In particular, we show that there are four different boundary conditions that preserve the triplet algebra, and check the consistency of the corresponding boundary operators by constructing their OPE coefficients explicitly. We also compute the correlation functions of two bulk fields in the presence of a boundary, and verify that they are consistent with factorisation.
\end{abstract}

\section{Introduction}

'Rational' logarithmic conformal field theories are logarithmic conformal field theories that behave in many respects like ordinary rational conformal field theories. They are, however, not rational in the strict sense since they contain indecomposable representations (that typically lead to correlation functions with logarithmic branch cuts). As such these theories provide an interesting class of models that allow one to probe how far methods developed for standard rational conformal field theory may in fact be applicable in a wider context. In this paper we study the boundary theory for one such logarithmic theory in detail, the 'rational' triplet theory at $c=-2$. As we shall see, certain aspects of our construction work as in the usual rational case, but there are also interesting differences.

The first example of a (non-rational) logarithmic conformal field theory was already found some time ago in 11] (see also [2]), and the first 'rational' example (that shall also concern us in this paper) was constructed in 3]; for some recent reviews see [4, 5, 6]. From a physics point of view, logarithmic conformal field theories appear naturally in various models of

*Email: gaberdiel@itp.phys.ethz.ch

${ }^{\dagger}$ Email: ingo.runkel@kcl.ac.uk 
statistical physics, for example in the theory of (multi)critical polymers [7, 8, 9], percolation [10, 11, and various critical (disordered) models [12, 13, 14, 15, 16, 17, 18, 19, 20]; a family of integrable lattice models with logarithmic critical behaviour has also recently been found in 21. There have been applications in string theory, in particular in the context of D-brane recoil [22, 23, 24, 25], and in pp-wave backgrounds [26]. Logarithmic vertex operator algebras have finally attracted some attention in mathematics [27, 28, 29]. Most work has been done on the $c=-2$ triplet theory, but logarithmic conformal field theories have also appeared in other contexts, in particular for theories with super group symmetries, see for example [2, 13, 30, 31, as well as in other classes of models, for example [32, 33, 34, 35].

Many structural aspects of logarithmic conformal field theories have been studied in detail, but there are still a number of issues that have not yet been satisfactorily understood. One concerns the structure of the boundary theory that is of some importance since lattice calculations typically involve boundaries (see for example [36, 21, 20]). Little is known in general about the structure of a logarithmic boundary theory [37, 38, 39, 40, and even for the simplest 'rational' theory, the triplet theory at $c=-2$, the situation is somewhat unclear. Various attempts to analyse the boundary conditions for this theory have been made in the past [38, 39, 40, 16, 41, 42, but these are partially conflicting and no clear consensus seems to have emerged.

In this paper we remedy this situation by studying the boundary theory of the $c=-2$ triplet model from first principles. We begin by constructing the boundary states using the free fermionic formulation of the theory, and find agreement with the boundary states of [40] and [16. These boundary states satisfy the Cardy condition, and we can read off from this analysis the boundary field content on the various boundaries and between different boundary conditions. With this information one can then analyse whether these boundary fields define indeed a consistent associative algebra. This amounts to constructing the relevant boundary OPE coefficients that have to satisfy the usual crossing relations; for the most interesting boundary fields (namely those that are primary with respect to the free fermion modes) we construct these OPE coefficients explicitly. We also determine the leading bulk-boundary OPE coefficients; this allows us to verify that our boundary conditions satisfy some of the factorisation constraints, i.e. that they are in fact compatible with the bulk theory of [43]. Many of these checks are highly non-trivial, and taken together they give very strong support to the assertion that the boundary conditions we construct are in fact consistent. At least for this logarithmic triplet theory we have therefore constructed the boundary theory in some detail. It would be interesting to understand which of the features of our construction will generalise for other 'rational' logarithmic theories.

The paper is organised as follows. In the next section we give a brief self-contained summary of our results. The boundary states are constructed in section 3 where we also determine the open string spectra. In section 4 we study the associativity of the boundary operators and construct the relevant OPE coefficients explicitly. The bulk-boundary OPE coefficients are determined in section 5 where we also use them to check the factorisation constraint involving two bulk fields on the upper half plane. Section 6 contains our conclusions and a brief outlook. Many of the technical details of our calculations (as well as some of the more mathematical subtleties) are explained in various appendices. 


\section{Summary of results}

Before explaining the detailed construction of the boundary theory for the logarithmic $c=-2$ model, let us briefly summarise our main findings. In order to be self-contained we begin by reviewing the structure of the bulk theory, following [44, 8, 9, 3, 43, 45].

\subsection{The chiral structure}

The symmetry algebra (or chiral algebra) of our theory is the triplet algebra that was defined in [44. It is generated by the Virasoro modes $L_{n}$, and the modes of a triplet of weight 3 fields $W_{n}^{a}$. The commutation relations are given in appendix $\mathrm{A}$

\subsubsection{Symplectic fermions}

The algebra has a free field realisation in terms of a pair of symplectic fermions $\chi^{\alpha}$ with $\alpha= \pm$ [43, 45]; these are fermionic fields of conformal weight $h=1$, whose anti-commutation relations are

$$
\left\{\chi_{m}^{\alpha}, \chi_{n}^{\beta}\right\}=m d^{\alpha \beta} \delta_{m,-n} .
$$

Here the anti-symmetric tensor $d^{\alpha \beta}$ is normalised to $d^{ \pm \mp}= \pm 1$. We also introduce the inverse tensor by $d_{\mp \pm}= \pm 1$. The triplet generators can be expressed in terms of these fermions as

$$
L_{-2} \Omega=\frac{1}{2} d_{\alpha \beta} \chi_{-1}^{\alpha} \chi_{-1}^{\beta} \Omega \quad, \quad W_{-3}^{a} \Omega=t_{\alpha \beta}^{a} \chi_{-2}^{\alpha} \chi_{-1}^{\beta} \Omega
$$

where $\Omega$ is the usual vacuum state that is annihilated by all $\chi_{m}^{\alpha} \Omega=0$ with $m \geq 0$, and the tensors $t_{\alpha \beta}^{a}$ are defined in (A.3. .

The triplet generators are bosonic generators, and the vacuum representation $\mathcal{H}_{\Omega}$ of the fermionic generators is therefore not irreducible with respect to the triplet algebra; instead we have

$$
\mathcal{H}_{\Omega}=\mathcal{V}_{0} \oplus \mathcal{V}_{1}
$$

Here $\mathcal{V}_{0}$ is the irreducible vacuum representation of the triplet algebra, and $\mathcal{V}_{1}$ is the highest weight representation whose highest weight states $\psi^{ \pm}=\chi_{-1}^{ \pm} \Omega$ form a doublet of states at conformal weight one (that are mapped into one another under the action of $W_{0}^{a}$ - see 3 ] for more details). In fact, $\mathcal{V}_{0}$ consists of all bosonic states in $\mathcal{H}_{\Omega}$, while $\mathcal{V}_{1}$ contains all the fermionic states.

\subsubsection{The relevant representations of the triplet algebra}

The vacuum representation $\mathcal{H}_{\Omega}$ is a subrepresentation of the (chiral) highest weight representation generated by $\omega$, where $\omega$ is characterised by $\chi_{m}^{\alpha} \omega=0$ for $m>0$ (but we do not assume that $\left.\chi_{0}^{\alpha} \omega=0\right)$. The space of ground states of this representation is four-dimensional; it consists of the two bosonic states $\omega$ and $\Omega=\chi_{0}^{-} \chi_{0}^{+} \omega$, as well as the two fermionic states

$\chi_{0}^{ \pm} \omega$. The representation $\mathcal{H}_{\omega}$ that is generated by the action of the free fermions from $\omega$ decomposes into two indecomposable representations of the triplet algebra

$$
\mathcal{H}_{\omega}=\mathcal{R}_{0} \oplus \mathcal{R}_{1}
$$


where $\mathcal{R}_{0}$ consists of the bosonic, and $\mathcal{R}_{1}$ of the fermionic states. The cyclic state of $\mathcal{R}_{0}$ is the highest weight state $\omega$; it is annihilated by all positive triplet modes as well as $W_{0}^{a}$, but satisfies

$$
L_{0} \omega=\Omega \text {. }
$$

It therefore defines an indecomposable but reducible highest weight representation of the triplet algebra [46, 3]. The representation $\mathcal{R}_{1}$ on the other hand is generated by the action of the triplet generators from a cyclic state at $h=1$ that is not highest weight; its structure is described in detail in appendix $\mathrm{A}$.

The other free fermion representation that is of relevance is the $\mathbb{Z}_{2}$ twisted representation that is generated from a highest weight state $\mu$ of conformal dimension $h=-1 / 8$ by the action of the half-integer moded symplectic fermions. (Since the triplet generators are bilinear in the symplectic fermions, these are the only two fermionic representations that lead to untwisted representations of the triplet algebra.) In terms of the triplet algebra, the representation $\mathcal{H}_{\mu}$ generated from $\mu$ decomposes into the two irreducible triplet representations

$$
\mathcal{H}_{\mu}=\mathcal{V}_{-1 / 8} \oplus \mathcal{V}_{3 / 8}
$$

These are conventional highest weight representations whose highest weight states have conformal weight $-1 / 8$ and $3 / 8$, respectively. The highest weight states of $\mathcal{V}_{3 / 8}$ are the doublet

$\chi_{-1 / 2}^{ \pm} \mu$ that are mapped into one another under the action of the $W_{0}^{a}$ modes - for details see [3].

It is also known that the triplet algebra is $C_{2}$-cofinite [3, 47, 48] and thus is 'rational' in that it has only finitely many highest weight representations. We mention in passing that the algebra possesses also other representations than the ones discussed above [49, 50]; however these additional representations do not appear in the state spaces of the triplet theory (for the cylinder as well as for the strip), and we need not discuss them here.

\section{$2.2 \quad$ The local theory}

We can construct a consistent local theory out of these representations 43. The space of states can best be described in terms of the fermionic description of the theory. To this end we consider the representations of the free fermions

$$
\mathcal{H}_{\omega} \equiv \mathcal{H}_{\omega} \otimes \overline{\mathcal{H}}_{\omega}, \quad \mathcal{H}_{\boldsymbol{\mu}} \equiv \mathcal{H}_{\mu} \otimes \overline{\mathcal{H}}_{\mu},
$$

where the barred spaces refer to the anti-chiral degrees of freedom. In both cases we then restrict to the bosonic degrees of freedom

$$
\begin{aligned}
& \mathcal{H}_{\omega}^{\text {bos }}=\left(\mathcal{R}_{0} \otimes \overline{\mathcal{R}}_{0}\right) \oplus\left(\mathcal{R}_{1} \otimes \overline{\mathcal{R}}_{1}\right) \\
& \mathcal{H}_{\boldsymbol{\mu}}^{\text {bos }}=\left(\mathcal{V}_{-1 / 8} \otimes \overline{\mathcal{V}}_{-1 / 8}\right) \oplus\left(\mathcal{V}_{3 / 8} \otimes \overline{\mathcal{V}}_{3 / 8}\right) .
\end{aligned}
$$

The total space of the local theory is finally

$$
\mathcal{H}^{\text {bulk }}=\mathcal{H}_{\omega}^{\text {bos }} / \mathcal{N} \oplus \mathcal{H}_{\mu}^{\text {bos }},
$$


where $\mathcal{N}$ is the subrepresentation (with respect to the chiral- and anti-chiral triplet algebra) of $\mathcal{H}_{\omega}^{\text {bos }}$ that is spanned by all states of the form

$$
\left(\chi_{0}^{\alpha}-\bar{\chi}_{0}^{\alpha}\right) \boldsymbol{\rho}
$$

with $\boldsymbol{\rho}$ an arbitrary vector in the fermionic subspace of $\mathcal{H}_{\boldsymbol{\omega}}$. The quotienting by $\mathcal{N}$ is necessary in order to obtain a local theory; in the quotient space we then have [43]

$$
L_{0} \boldsymbol{\omega}=\Omega \otimes \bar{\omega}+\mathcal{N}=\omega \otimes \bar{\Omega}+\mathcal{N}=\bar{L}_{0} \boldsymbol{\omega}
$$

since

$$
\Omega \otimes \bar{\omega}-\omega \otimes \bar{\Omega}=\left[\left(\chi_{0}^{-}-\bar{\chi}_{0}^{-}\right) \chi_{0}^{+}-\left(\chi_{0}^{+}-\bar{\chi}_{0}^{+}\right) \bar{\chi}_{0}^{-}\right] \omega \otimes \bar{\omega} \in \mathcal{N} .
$$

Here $\boldsymbol{\omega}$ denotes the equivalence class that contains $\omega \otimes \bar{\omega}$. The other highest weight state of $\mathcal{H}_{\omega}^{\text {bos }} / \mathcal{N}$ will be denoted by

$$
\Omega=\omega \otimes \bar{\Omega}+\mathcal{N}=\Omega \otimes \bar{\omega}+\mathcal{N}
$$

(Note that $\Omega \otimes \Omega \in \mathcal{N}$ since it is equal to $\left(\chi_{0}^{-}-\bar{\chi}_{0}^{-}\right) \bar{\chi}_{0}^{-} \bar{\chi}_{0}^{+} \chi_{0}^{+} \boldsymbol{\omega}$.) In terms of the triplet generators the resulting representation space is then quite complicated [43: given the decomposition (2.4) it contains states from $\mathcal{R}_{0} \otimes \overline{\mathcal{R}}_{0}$ and $\mathcal{R}_{1} \otimes \overline{\mathcal{R}}_{1}$, but these get partially identified upon quotienting by $\mathcal{N}$.

\subsubsection{Amplitudes and operator product expansions}

In the following we shall mainly consider the amplitudes of the fields $\boldsymbol{\omega}$ and $\boldsymbol{\mu}$; the amplitudes for the other fields can be obtained from these using the fermionic symmetry. We choose the conventions $^{1}$

$$
\langle\boldsymbol{\omega}(z)\rangle=-1 \quad, \quad\left\langle\boldsymbol{\omega}\left(z_{1}\right) \boldsymbol{\omega}\left(z_{2}\right)\right\rangle=4 \log \left|z_{1}-z_{2}\right| .
$$

The leading terms in the operator product expansion of the fields $\boldsymbol{\omega}$ and $\boldsymbol{\mu}$ are then [43]

$$
\begin{aligned}
& \boldsymbol{\omega}(z) \boldsymbol{\omega}(0)=-4 \log |z|(\boldsymbol{\omega}(0)+\log |z| \boldsymbol{\Omega}(0))+\cdots \\
& \boldsymbol{\mu}(z) \boldsymbol{\omega}(0)=-2(2 \log 2+\log |z|) \boldsymbol{\mu}(0)+\cdots \\
& \boldsymbol{\mu}(z) \boldsymbol{\mu}(0)=|z|^{\frac{1}{2}}(-\boldsymbol{\omega}(0)+2(2 \log 2-\log |z|) \boldsymbol{\Omega}(0)+\cdots) .
\end{aligned}
$$

\subsection{The boundary theory}

As we shall explain in more detail below, the above triplet theory has four different boundary states that respect the full triplet symmetry. All four of them can be most easily described

\footnotetext{
${ }^{1}$ This is different from 43: if we denote the fields from 43 by $\boldsymbol{\omega}^{\mathrm{GK}}, \boldsymbol{\Omega}^{\mathrm{GK}}$ and $\boldsymbol{\mu}^{\mathrm{GK}}$, the relation to the fields used here is $\boldsymbol{\omega}=\boldsymbol{\omega}^{\mathrm{GK}}+4 \log 2 \boldsymbol{\Omega}^{\mathrm{GK}}, \boldsymbol{\Omega}=\boldsymbol{\Omega}^{\mathrm{GK}}$ and $\boldsymbol{\mu}=i \boldsymbol{\mu}^{\mathrm{GK}}$. We also choose $\mathcal{C}_{0}=-1$. With these conventions the 2-point function of $\boldsymbol{\omega}$ is particularly simple, and the bulk-boundary OPE coefficients of $\boldsymbol{\mu}$, as well as the coefficients of the boundary states, are real.
} 
in the free fermion language: there are two 'Neumann' boundary states, $\| N, \pm\rangle\rangle$, that satisfy the gluing conditions

$$
\left.\left.\left(\chi_{n}^{\alpha}+\bar{\chi}_{-n}^{\alpha}\right) \| \mathrm{N}, \pm\right\rangle\right\rangle=0,
$$

as well as two 'Dirichlet' boundary states, $\| \mathrm{D}, \pm\rangle\rangle$, that are characterised by

$$
\left.\left(\chi_{n}^{\alpha}-\bar{\chi}_{-n}^{\alpha}\right) \| \mathrm{D}, \pm\right\rangle=0 .
$$

The relevant open string spectra are (see section 3i)

$$
\begin{aligned}
\left\langle\left\langle\mathrm{N}, \pm\left\|q^{L_{0}+\bar{L}_{0}-\frac{c}{12}}\right\| \mathrm{N}, \pm\right\rangle\right\rangle & =\chi_{\mathcal{R}_{0}}(\tilde{q}) \\
\left\langle\left\langle\mathrm{N}, \pm\left\|q^{L_{0}+\bar{L}_{0}-\frac{c}{12}}\right\| \mathrm{N}, \mp\right\rangle\right\rangle & =\chi_{\mathcal{R}_{1}}(\tilde{q}) \\
\left\langle\left\langle\mathrm{D}, \pm\left\|q^{L_{0}+\bar{L}_{0}-\frac{c}{12}}\right\| \mathrm{D}, \pm\right\rangle\right\rangle & =\chi_{\mathcal{V}_{0}}(\tilde{q}) \\
\left\langle\left\langle\mathrm{D}, \pm\left\|q^{L_{0}+\bar{L}_{0}-\frac{c}{12}}\right\| \mathrm{D}, \mp\right\rangle\right\rangle & =\chi_{\mathcal{V}_{1}}(\tilde{q}) \\
\left\langle\left\langle\mathrm{N}, \pm\left\|q^{L_{0}+\bar{L}_{0}-\frac{c}{12}}\right\| \mathrm{D}, \pm\right\rangle\right\rangle & =\chi_{\mathcal{V}_{-1 / 8}}(\tilde{q}) \\
\left\langle\left\langle\mathrm{N}, \pm\left\|q^{L_{0}+\bar{L}_{0}-\frac{c}{12}}\right\| \mathrm{D}, \mp\right\rangle\right\rangle & =\chi_{\mathcal{V}_{3 / 8}}(\tilde{q})
\end{aligned}
$$

where $\tilde{q}$ is the open string loop parameter. These spectra agree with what was found in [40] (see also [16]). In fact, we may identify our boundary states with the four irreducible representations as follows

$$
\begin{array}{ll}
\left.\left.\left.\left.\| \mathcal{V}_{0}\right\rangle\right\rangle=\| \mathrm{D},+\right\rangle\right\rangle & \left.\left.\left.\left.\| \mathcal{V}_{1}\right\rangle\right\rangle=\| \mathrm{D},-\right\rangle\right\rangle \\
\left.\left.\left.\left.\| \mathcal{V}_{-1 / 8}\right\rangle\right\rangle=\| \mathrm{N},+\right\rangle\right\rangle & \left.\left.\left.\left.\| \mathcal{V}_{3 / 8}\right\rangle\right\rangle=\| \mathrm{N},-\right\rangle\right\rangle .
\end{array}
$$

The open string spectra are then simply given by the fusion rules

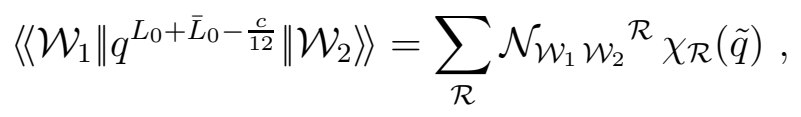

where $\mathcal{N}$ denotes the fusion rules that were calculated in [3]. Here $\mathcal{W}_{i}$ denotes an irreducible representation, while $\mathcal{R}$ runs over all indecomposable representations. This description of the boundary states is therefore very reminiscent of the usual rational case.

The boundary operators that live on each of the two Dirichlet boundary conditions lie in the irreducible vacuum representation $\mathcal{V}_{0}$ with highest weight state $\Omega$. For the two Neumann boundary conditions, on the other hand, the boundary operators lie in the reducible representation $\mathcal{R}_{0}$ whose ground states are $\Omega$ and $\omega$. We choose again the convention that $L_{0} \omega=\Omega$, and that $\Omega$ acts as the identity field in boundary correlators. Furthermore, we choose our normalisation so that

$$
\langle\omega(x) \omega(y)\rangle_{\mathrm{uhp}}^{\mathrm{N}, \pm}=-2 \log (x-y)\langle\omega(0)\rangle_{\mathrm{uhp}}^{\mathrm{N}, \pm},
$$

where $x$ and $y$ lie on the real axis that describes the boundary of the upper half plane (uhp). We have determined the leading order in the boundary operator product expansion

$$
\omega(x) \omega(0)=-(\log x)^{2} \Omega(0)-2 \log x \omega(0)+\cdots,
$$


and we check in section 4 that these boundary operators define indeed an associative algebra. We also confirm there the consistency with the operator product expansions of boundary changing operators $\mu$ that interpolate between $(\mathrm{D}, \pm)$ and $(\mathrm{N}, \pm)$ boundaries.

Finally we have determined the bulk-boundary operator product expansions, and we have found that on the upper half plane the resulting expressions are

$$
\begin{aligned}
\left.\boldsymbol{\mu}(i y)\right|_{\mathrm{D}, \eta} & =\eta \sqrt{\pi}(2 y)^{\frac{1}{4}} \Omega(0)+\ldots \\
\left.\boldsymbol{\mu}(i y)\right|_{\mathrm{N}, \eta} & =-\eta \frac{2}{\sqrt{\pi}}(2 y)^{\frac{1}{4}}(\omega(0)+(\log (2 y)-2 \log 2) \Omega(0))+\ldots \\
\left.\boldsymbol{\omega}(i y)\right|_{\mathrm{D}, \eta} & =-2 \log (2 y) \Omega(0)+\ldots \\
\left.\boldsymbol{\omega}(i y)\right|_{\mathrm{N}, \eta} & =4 \omega(0)+2 \log (2 y) \Omega(0)+\ldots
\end{aligned}
$$

where $\eta= \pm$. We have checked that with these conventions, the boundary conditions satisfy the factorisation constraints that come from considering the amplitude with two bulk fields on the upper half plane (see section 5).

Given that our boundary conditions satisfy all of these consistency conditions, it is very plausible to believe that they are indeed consistent boundary conditions of the triplet bulk theory. Thus, at least for this logarithmic theory, we have managed to construct the boundary theory.

The rest of the paper is somewhat more technical; in the following sections we shall explain in detail how to derive these results and check their consistency. The first step is the construction of the boundary states.

\section{The construction of the boundary states}

\subsection{Ishibashi states}

In the following we want to construct all the boundary states of the triplet theory that preserve the triplet symmetry (with trivial gluing automorphism). These boundary states have to lie in the subspace $B$ of (a suitable completion of) $\mathcal{H}^{\text {bulk }}$ given by

$$
\left.\left.\left.\left.B=\{|v\rangle\rangle \in \mathcal{H}^{\text {bulk }}\left|\left(L_{m}-\bar{L}_{-m}\right)\right| v\right\rangle\right\rangle=0=\left(W_{m}^{a}+\bar{W}_{-m}^{a}\right)|v\rangle\right\rangle ; m \in \mathbb{Z}, a \in\{+, 0,-\}\right\}
$$

For usual rational conformal field theories whose space of states is a direct sum of tensor products $\mathcal{H}_{i} \otimes \overline{\mathcal{H}}_{j}$ where $\mathcal{H}_{i}$ and $\overline{\mathcal{H}}_{j}$ are irreducible representations, the space of solutions is spanned by the Ishibashi states, of which there is (up to normalisation) one in each sector $\mathcal{H}_{i} \otimes \overline{\mathcal{H}}_{i}$. For the triplet theory two new features appear: some of the representations are indecomposable but reducible, and the space of states is actually a quotient. A sector by sector analysis of possible solutions to the conditions in (3.1) (without imposing any constraints that come from the quotient space $\mathcal{N}$ ) has been carried out in 41]; the results have also been compared to what one obtains from requiring the fermionic symmetries to be preserved 42. 
In the following we shall not try to construct the space $B \subset \mathcal{H}^{\text {bulk }}$ directly, but first consider the Ishibashi states that preserve the full fermionic symmetries. The relevant gluing conditions for the fermions that guarantee that the triplet symmetry is preserved are either 'Dirichlet'

$$
\left.\left.\left(\chi_{n}^{ \pm}-\bar{\chi}_{-n}^{ \pm}\right) \| \mathrm{D}\right\rangle\right\rangle=0
$$

or 'Neumann'

$$
\left.\left.\left(\chi_{n}^{ \pm}+\bar{\chi}_{-n}^{ \pm}\right) \| \mathrm{N}\right\rangle\right\rangle=0
$$

where it is understood that $n \in \mathbb{Z}$ for the sector $\mathcal{H}_{\omega}^{\text {bos }} / \mathcal{N} \subset \mathcal{H}^{\text {bulk }}$, while $n \in \mathbb{Z}+\frac{1}{2}$ for $\mathcal{H}_{\mu}^{\text {bos }}$.

Given a state $\boldsymbol{\rho} \in \mathcal{H}^{\text {bulk }}$ that is annihilated by all positive fermion modes, the coherent states

$$
\begin{aligned}
& |\boldsymbol{\rho}\rangle\rangle^{(\mathrm{D})}=\exp \left(\sum_{n>0} \frac{1}{n}\left(\chi_{-n}^{-} \bar{\chi}_{-n}^{+}-\chi_{-n}^{+} \bar{\chi}_{-n}^{-}\right)\right) \boldsymbol{\rho} \\
& |\boldsymbol{\rho}\rangle\rangle^{(\mathrm{N})}=\exp \left(-\sum_{n>0} \frac{1}{n}\left(\chi_{-n}^{-} \bar{\chi}_{-n}^{+}-\chi_{-n}^{+} \bar{\chi}_{-n}^{-}\right)\right) \boldsymbol{\rho}
\end{aligned}
$$

satisfy the conditions (3.2) or (3.3), respectively, for $n \neq 0$. Since there are no fermionic zero modes in $\mathcal{H}_{\boldsymbol{\mu}}^{\text {bos }}$, we therefore have two solutions to (3.1) by taking $\boldsymbol{\rho}=\boldsymbol{\mu}$.

The analysis is more complicated if $\boldsymbol{\rho} \in \mathcal{H}_{\omega}^{\text {bos }} / \mathcal{N}$, since there are zero modes, and we need to impose (3.2) and (3.3) also for these zero modes. ${ }^{2}$ The zero mode constraint of (3.2) is trivial since the image under $\chi_{0}^{ \pm}-\bar{\chi}_{0}^{ \pm}$lies in $\mathcal{N}$, and is thus automatically zero in the quotient space. On the other hand, it is clear that $\boldsymbol{\omega}$ does not satisfy the zero mode constraint of (3.3) since $\left(\chi_{0}^{ \pm}+\bar{\chi}_{0}^{ \pm}\right) \boldsymbol{\omega}=2 \chi_{0}^{ \pm} \boldsymbol{\omega} \neq 0$. (There is no such condition for the Ishibashi states based on $\Omega$, since $\Omega$ is annihilated by all fermionic zero modes.) Thus (3.4) gives only three fermionic Ishibashi states in the sector $\mathcal{H}_{\omega}^{\text {bos }} / \mathcal{N} \subset \mathcal{H}^{\text {bulk }}$. So far, we therefore have the five Ishibashi states

$$
\begin{aligned}
& \text { (D) : } \left.\left.\quad|\boldsymbol{\mu}\rangle\rangle^{(\mathrm{D})} \quad|\boldsymbol{\omega}\rangle\right\rangle^{(\mathrm{D})} \quad|\boldsymbol{\Omega}\rangle\right\rangle^{(\mathrm{D})} \\
& \left.(\mathrm{N}): \quad|\boldsymbol{\mu}\rangle\rangle^{(\mathrm{N})} \quad|\boldsymbol{\Omega}\rangle\right\rangle^{(\mathrm{N})} .
\end{aligned}
$$

The space $B$ thus has at least dimension five. We now proceed to prove that it has exactly dimension five, i.e. that the Ishibashi states (3.5) $\operatorname{span} B$.

Every element $|v\rangle\rangle$ of $B$ can be written as a direct sum $\left.\left.|v\rangle\rangle=\left|v_{\omega}\right\rangle\right\rangle \oplus\left|v_{\mu}\right\rangle\right\rangle$, where $\left.\left|v_{\omega}\right\rangle\right\rangle \in$ $\mathcal{H}_{\omega}^{\text {bos }} / \mathcal{N}$ and $\left.\left|v_{\mu}\right\rangle\right\rangle \in \mathcal{H}_{\mu}^{\text {bos }}$. Since the action of $L_{m}$ and $W_{m}^{a}$ does not mix these two sectors, it follows that also $\left.\left|v_{\omega}\right\rangle\right\rangle$ and $\left.\left|v_{\mu}\right\rangle\right\rangle$ must be individually in $B$. It is therefore enough to look for solutions to (3.1) in these two sectors of $\mathcal{H}^{\text {bulk }}$ separately. Accordingly we will write $B=B_{\omega} \oplus B_{\mu}$.

Let us start with $\mathcal{H}_{\boldsymbol{\mu}}^{\text {bos }}$. Recall that this sector decomposes as $\left(\mathcal{V}_{-1 / 8} \otimes \overline{\mathcal{V}}_{-1 / 8}\right) \oplus\left(\mathcal{V}_{3 / 8} \otimes \overline{\mathcal{V}}_{3 / 8}\right)$ into triplet representations. These are all irreducible, and so this sector gives rise to exactly two Ishibashi states. We have already found two states in $B_{\mu}$, namely $\left.|\boldsymbol{\mu}\rangle\right\rangle^{(\mathrm{D})}$ and $\left.|\boldsymbol{\mu}\rangle\right\rangle^{(\mathrm{N})}$, and hence these already span $B_{\mu}$ (each of these two states is a linear combinations of the Ishibashi states corresponding to the two triplet representations).

\footnotetext{
${ }^{2}$ This seems to have been overlooked in [0].
} 
The analysis in the sector $\mathcal{H}_{\boldsymbol{\omega}}^{\text {bos }} / \mathcal{N}$ is more involved. We already know that the three states $\left.|\boldsymbol{\omega}\rangle\rangle^{(\mathrm{D})},|\boldsymbol{\Omega}\rangle\right\rangle^{(\mathrm{D})}$ and $\left.|\boldsymbol{\Omega}\rangle\right\rangle^{(\mathrm{N})}$ lie in $B_{\omega}$. Let now $\left.|b\rangle\right\rangle$ be an arbitrary element in $B_{\omega}$. We can expand $|b\rangle\rangle$ as

$$
|b\rangle\rangle=\sum_{k=0}^{\infty} v^{(k)} ; \quad v^{(k)} \text { has grade }(k, k) .
$$

In the quotient space $\mathcal{H}_{\omega}^{\text {bos }} / \mathcal{N}$, we can replace any zero mode $\bar{\chi}_{0}^{a}$ by $\chi_{0}^{a}$. Then it is easy to see that the two lowest grades have to be of the form

$$
v^{(0)}=b^{\omega} \boldsymbol{\omega}+b^{\Omega} \boldsymbol{\Omega}, \quad v^{(1)}=b_{\alpha \beta}^{\omega} \chi_{-1}^{\alpha} \bar{\chi}_{-1}^{\beta} \boldsymbol{\omega}+b_{\alpha \beta}^{\Omega} \chi_{-1}^{\alpha} \bar{\chi}_{-1}^{\beta} \boldsymbol{\Omega}
$$

for some constants $b^{\omega}, b^{\Omega}, b_{\alpha \beta}^{\omega}$, and $b_{\alpha \beta}^{\Omega}$. Consider the following linear combination of Ishibashi states,

$$
\left.\left.\left.\left.\left.\left.\left.\left|b^{\prime}\right\rangle\right\rangle=|b\rangle\right\rangle-b^{\omega}|\boldsymbol{\omega}\rangle\right\rangle^{(\mathrm{D})}-\frac{1}{2} b^{\Omega}(|\boldsymbol{\Omega}\rangle\rangle^{(\mathrm{D})}+|\boldsymbol{\Omega}\rangle\right\rangle^{(\mathrm{N})}\right)-\frac{1}{2} b_{-+}^{\Omega}(|\boldsymbol{\Omega}\rangle\rangle^{(\mathrm{D})}-|\boldsymbol{\Omega}\rangle\right\rangle^{(\mathrm{N})}\right) .
$$

We will prove below that $\left.\left|b^{\prime}\right\rangle\right\rangle=0$ and hence that every element in $B_{\omega}$ is a linear combination of the three fermionic states already found. This then implies $\operatorname{dim} B_{\omega}=3$, as claimed.

Let us decompose $\left.\left|b^{\prime}\right\rangle\right\rangle=\sum_{k=0}^{\infty} u^{(k)}$, where again $u^{(k)}$ has grade $(k, k)$. The linear combination (3.8) is chosen such that $u^{(0)}=0$. We proceed by an induction argument. Suppose we know that $u^{(k)}=0$ for $k \leq N-1$ for some $N>0$. Then the conditions in (3.1) imply, for $m>0$,

$$
L_{m} u^{(N)}=\bar{L}_{-m} u^{(N-2 m)}=0, \quad \bar{L}_{m} u^{(N)}=L_{-m} u^{(N-2 m)}=0,
$$

where in each chain of equalities, the first equality follows since $\left.\left(L_{m}-\bar{L}_{-m}\right)\left|b^{\prime}\right\rangle\right\rangle=0$, while the second follows from $u^{(k)}=0$ for $k<N$. Similarly one sees that $W_{m}^{a} u^{(N)}=0=\bar{W}_{m}^{a} u^{(N)}$. Thus $u^{(N)}$ must be a triplet primary. The only triplet primary states in $\mathcal{H}_{\omega}^{\text {bos }} / \mathcal{N}$ are at grades $^{3}(0,0),(1,0),(0,1)$ and $(1,1)$. Thus we only need to consider the case $N=1$, for which $u^{(1)}$ is given by

$$
u^{(1)}=f_{\alpha \beta}^{\omega} \chi_{-1}^{\alpha} \bar{\chi}_{-1}^{\beta} \boldsymbol{\omega}+f_{\alpha \beta}^{\Omega} \chi_{-1}^{\alpha} \bar{\chi}_{-1}^{\beta} \Omega ; \quad f_{\alpha \beta}^{\omega}=b_{\alpha \beta}^{\omega}-b^{\omega} d_{\alpha \beta}, \quad f_{\alpha \beta}^{\Omega}=b_{\alpha \beta}^{\Omega}-b_{-+}^{\Omega} d_{\alpha \beta} .
$$

By construction we have $f_{-+}^{\Omega}=0$. Since the action of $L_{1}, W_{1}^{a}, \bar{L}_{1}, \bar{W}_{1}^{a}$ on a grade $(1,1)$ state contributes one zero mode, we see that $\chi_{-1}^{\alpha} \bar{\chi}_{-1}^{\beta} \Omega$ is triplet primary. Similarly, one can also check that $\chi_{-1}^{\alpha} \bar{\chi}_{-1}^{\beta} \boldsymbol{\omega}$ is not triplet primary. It therefore follows that $f_{\alpha \beta}^{\omega}=0$. Finally, we impose the zero mode condition $\left(W_{0}^{a}+\bar{W}_{0}^{a}\right) u^{(1)}=0$. In terms of fermion modes, this is equivalent to demanding

$$
t_{\rho \sigma}^{a}\left(\chi_{-1}^{\rho} \chi_{1}^{\sigma}+\bar{\chi}_{-1}^{\rho} \bar{\chi}_{1}^{\sigma}\right) f_{\alpha \beta}^{\Omega} \chi_{-1}^{\alpha} \bar{\chi}_{-1}^{\beta} \Omega=0 .
$$

Expressing this in terms of negative fermion modes only, one finds that (3.11) is equivalent to $t_{\gamma \sigma}^{a} d^{\sigma \alpha} f_{\alpha \delta}^{\Omega}+t_{\delta \sigma}^{a} d^{\sigma \beta} f_{\gamma \beta}^{\Omega}=0$ for all $a=0, \pm$ and $\gamma, \delta \in\{ \pm\}$. This in turn can be reduced to

\footnotetext{
${ }^{3}$ This is clear for the representation $\mathcal{H}_{\omega}^{\text {bos }}=\left(\mathcal{R}_{0} \otimes \mathcal{R}_{0}\right) \oplus\left(\mathcal{R}_{1} \otimes \mathcal{R}_{1}\right)$, but some care has to be taken for the quotient $\mathcal{H}_{\boldsymbol{\omega}}^{\text {bos }} / \mathcal{N}$. Note however, that $\mathcal{H}_{\boldsymbol{\omega}}^{\text {bos }} / \mathcal{N}$ is in particular a representation of the holomorphic copy of the triplet algebra in the bulk. All its representations (with integer generalised $L_{0}$-weights) are known, and they have highest weight states only for grades 0 or 1 . The same applies to the anti-holomorphic copy of the triplet algebra.
} 
$f_{++}^{\Omega}=f_{--}^{\Omega}=0$ and $f_{+-}^{\Omega}+f_{-+}^{\Omega}=0$. As we have already found that $f_{-+}^{\Omega}=0$ we can conclude that $f_{\alpha \beta}^{\Omega}=0$. Thus also $u^{(1)}=0$. Altogether we therefore find that indeed $\left.\left|b^{\prime}\right\rangle\right\rangle=0$.

This completes the proof that the five Ishibashi states (3.5) are a basis of $B$. Naively, we would therefore expect that there are five boundary states for the triplet theory. However, as we shall see, there are only four linear combinations that actually define boundary conditions that are consistent with the Cardy constraint.

\subsection{Calculation of cylinder diagrams}

In order to determine the consistent boundary states we can use the Cardy condition. This requires calculating the cylinder amplitude between the different Ishibashi states given above. For the logarithmic theory we are considering here, there is a subtlety regarding the definition of the inner product between states, and we therefore need to explain carefully what we need to calculate.

As is explained in appendix C. every conformal field theory (logarithmic or not) possesses a bilinear form on the bulk space; the cylinder amplitude is then simply the bilinear form evaluated on the two boundary states, i.e.

$$
\left.\left.\left.\left.\left\langle\left\langle\mathrm{B}_{1}\left\|q^{\frac{1}{2}\left(L_{0}+\bar{L}_{0}\right)-\frac{c}{24}}\right\| \mathrm{B}_{2}\right\rangle\right\rangle=B\left(\| \mathrm{B}_{1}\right\rangle\right\rangle, q^{\frac{1}{2}\left(L_{0}+\bar{L}_{0}\right)-\frac{c}{24}} \| \mathrm{B}_{2}\right\rangle\right\rangle\right)
$$

With this prescription and the definitions of appendix $\mathbb{C}$ (recall that $B(\boldsymbol{\omega}, \boldsymbol{\Omega})=-1$ and $B(\boldsymbol{\mu}, \boldsymbol{\mu})=1$ ), it is then straightforward to calculate the overlaps between the Ishibashi states

$$
\begin{aligned}
& { }^{(\mathrm{D})}\left\langle\left\langle\boldsymbol{\mu}\left|q^{\frac{1}{2}\left(L_{0}+\bar{L}_{0}\right)-\frac{c}{24}}\right| \boldsymbol{\mu}\right\rangle\right\rangle^{(\mathrm{D})}={ }^{(\mathrm{N})}\left\langle\left\langle\boldsymbol{\mu}\left|q^{\frac{1}{2}\left(L_{0}+\bar{L}_{0}\right)-\frac{c}{24}}\right| \boldsymbol{\mu}\right\rangle\right\rangle^{(\mathrm{N})}=f_{4}(q)^{2}=f_{2}(\tilde{q})^{2} \\
& { }^{(\mathrm{D})}\left\langle\left\langle\boldsymbol{\mu}\left|q^{\frac{1}{2}\left(L_{0}+\bar{L}_{0}\right)-\frac{c}{24}}\right| \boldsymbol{\mu}\right\rangle\right\rangle^{(\mathrm{N})}={ }^{(\mathrm{N})}\left\langle\left\langle\boldsymbol{\mu}\left|q^{\frac{1}{2}\left(L_{0}+\bar{L}_{0}\right)-\frac{c}{24}}\right| \boldsymbol{\mu}\right\rangle\right\rangle^{(\mathrm{D})}=f_{3}(q)^{2}=f_{3}(\tilde{q})^{2} \\
& { }^{(\mathrm{D})}\left\langle\left\langle\boldsymbol{\omega}\left|q^{\frac{1}{2}\left(L_{0}+\bar{L}_{0}\right)-\frac{c}{24}}\right| \boldsymbol{\Omega}\right\rangle\right\rangle^{(\mathrm{D})}={ }^{(\mathrm{D})}\left\langle\left\langle\boldsymbol{\Omega}\left|q^{\frac{1}{2}\left(L_{0}+\bar{L}_{0}\right)-\frac{c}{24}}\right| \boldsymbol{\omega}\right\rangle\right\rangle^{(\mathrm{D})}=-f_{1}(q)^{2}=i \tilde{\tau} f_{1}(\tilde{q})^{2} \\
& { }^{(\mathrm{D})}\left\langle\left\langle\boldsymbol{\omega}\left|q^{\frac{1}{2}\left(L_{0}+\bar{L}_{0}\right)-\frac{c}{24}}\right| \boldsymbol{\Omega}\right\rangle\right\rangle^{(\mathrm{N})}={ }^{(\mathrm{N})}\left\langle\left\langle\boldsymbol{\Omega}\left|q^{\frac{1}{2}\left(L_{0}+\bar{L}_{0}\right)-\frac{c}{24}}\right| \boldsymbol{\omega}\right\rangle\right\rangle^{(\mathrm{D})}=-\frac{1}{2} f_{2}(q)^{2}=-\frac{1}{2} f_{4}(\tilde{q})^{2} \\
& \text { (D) }\left\langle\left\langle\boldsymbol{\omega}\left|q^{\frac{1}{2}\left(L_{0}+\bar{L}_{0}\right)-\frac{c}{24}}\right| \boldsymbol{\omega}\right\rangle\right\rangle^{(\mathrm{D})}=-2 \pi i \tau f_{1}(q)^{2}=2 \pi f_{1}(\tilde{q})^{2} \text {, }
\end{aligned}
$$

while all other overlaps vanish. Here $\tilde{q}=e^{2 \pi i \tilde{\tau}}$ is the open string loop parameter, with $\tilde{\tau}=-1 / \tau$. The relevant theta functions $f_{i}$, as well as their behaviour under modular transformations, are given in appendix B.

\subsection{Solution of Cardy condition}

Using the simple modular transformation properties stated in (3.13), it is easy to check that the following four boundary states satisfy the Cardy condition, i.e. give overlaps that can 
be interpreted as open string state spaces ( $c f .40$ ):

$$
\begin{aligned}
\| \mathrm{D}, \pm\rangle\rangle & \left.\left.=\frac{1}{2}|\boldsymbol{\mu}\rangle\right\rangle^{(\mathrm{D})} \mp \frac{1}{\sqrt{4 \pi}}|\boldsymbol{\omega}\rangle\right\rangle^{(\mathrm{D})} \\
\| \mathrm{N}, \pm\rangle\rangle & \left.=|\boldsymbol{\mu}\rangle\rangle^{(\mathrm{N})} \pm \sqrt{4 \pi}|\boldsymbol{\Omega}\rangle\right\rangle^{(\mathrm{N})}
\end{aligned}
$$

The overlaps of these branes are then

$$
\begin{aligned}
& \left\langle\left\langle\mathrm{D}, \pm\left\|q^{\frac{1}{2}\left(L_{0}+\bar{L}_{0}\right)-\frac{c}{24}}\right\| \mathrm{D}, \pm\right\rangle\right\rangle=\frac{1}{4} f_{2}(\tilde{q})^{2}+\frac{1}{2} f_{1}(\tilde{q})^{2}=\chi_{\mathcal{V}_{0}}(\tilde{q}) \\
& \left\langle\left\langle\mathrm{D}, \pm\left\|q^{\frac{1}{2}\left(L_{0}+\bar{L}_{0}\right)-\frac{c}{24}}\right\| \mathrm{D}, \mp\right\rangle\right\rangle=\frac{1}{4} f_{2}(\tilde{q})^{2}-\frac{1}{2} f_{1}(\tilde{q})^{2}=\chi_{\mathcal{V}_{1}}(\tilde{q}) \\
& \left\langle\left\langle\mathrm{N}, \pm\left\|q^{\frac{1}{2}\left(L_{0}+\bar{L}_{0}\right)-\frac{c}{24}}\right\| \mathrm{N}, \pm\right\rangle\right\rangle=f_{2}(\tilde{q})^{2}=\chi_{\mathcal{R}_{0}}(\tilde{q}) \\
& \left\langle\left\langle\mathrm{N}, \pm\left\|q^{\frac{1}{2}\left(L_{0}+\bar{L}_{0}\right)-\frac{c}{24}}\right\| \mathrm{N}, \mp\right\rangle\right\rangle=f_{2}(\tilde{q})^{2}=\chi_{\mathcal{R}_{1}}(\tilde{q}) \\
& \left\langle\left\langle\mathrm{D}, \pm\left\|q^{\frac{1}{2}\left(L_{0}+\bar{L}_{0}\right)-\frac{c}{24}}\right\| \mathrm{N}, \pm\right\rangle\right\rangle=\frac{1}{2} f_{3}(\tilde{q})^{2}+\frac{1}{2} f_{4}(\tilde{q})^{2}=\chi_{\mathcal{V}_{-1 / 8}}(\tilde{q}) \\
& \left\langle\left\langle\mathrm{D}, \pm\left\|q^{\frac{1}{2}\left(L_{0}+\bar{L}_{0}\right)-\frac{c}{24}}\right\| \mathrm{N}, \mp\right\rangle\right\rangle=\frac{1}{2} f_{3}(\tilde{q})^{2}-\frac{1}{2} f_{4}(\tilde{q})^{2}=\chi_{\mathcal{V}_{3 / 8}}(\tilde{q})
\end{aligned}
$$

where $\tilde{q}$ is the open string loop parameter. This is in agreement with the results of [40, 16] and reproduces our claim of section 2.3 . The character identities for the triplet representations were first derived in [8, 9].

Note that the identification of characters with representations is not unique; indeed one has to take into account the ambiguity

$$
\chi_{\mathcal{R}_{0}}(q)=\chi_{\mathcal{R}_{1}}(q)=2 \chi_{\mathcal{V}_{0}}(q)+2 \chi_{\mathcal{V}_{1}}(q) .
$$

Comparing with the overlaps (3.15) we see that the representations formed by the various open state spaces are uniquely determined by the characters in all cases except for the four overlaps involving only $(\mathrm{N}, \pm$ )-boundary conditions. In these cases the assignment of representations to the open state spaces as suggested by the notation used in (3.15) is an ansatz, which will be subjected to strong consistency checks in the following. We have also used that the overlap between $(\mathrm{N}, \eta)$ and $(\mathrm{N}, \eta)$ has opposite fermion number relative to that between $(\mathrm{N}, \eta)$ and $(\mathrm{N},-\eta)$; if one is to be identified with $\mathcal{R}_{0}$ the other must then be $\mathcal{R}_{1}$.

There are at least two ways to arrive at the boundary states (3.14). The first starts from the formulation of the boundary conditions in terms of symplectic fermions as in (3.2) and (3.3). One then demands the boundary states (rather than only the specific basis of Ishibashi states (3.5) to be compatible with these fermionic gluing conditions, which amounts to taking a boundary state to be either a linear combination of only the (D) Ishibashi states in (3.5), or of only the (N) Ishibashi states. Imposing the Cardy condition on this restricted ansatz, and demanding that the resulting set of boundary states cannot be written as a non-trivial non-negative integer combination of another set, one arrives at (3.14).

Second, as was done in [40], one can demand the existence of a boundary state $\left.\left.\| \mathcal{V}_{0}\right\rangle\right\rangle$, for which the space of open string states form the vacuum representation $\mathcal{V}_{0}$ of the triplet 
algebra. Finding a maximal set of fundamental boundary states compatible with $\left.\left.\| \mathcal{V}_{0}\right\rangle\right\rangle$ then also leads to (3.14).

However, this is not to say that (3.14) is the only set of boundary states consistent with the Cardy condition. For example, one can check that $\left.\left.|\boldsymbol{\mu}\rangle\rangle^{(\mathrm{D})}+\alpha_{k}|\boldsymbol{\Omega}\rangle\right\rangle^{(\mathrm{D})}+\beta_{k}|\boldsymbol{\Omega}\rangle\right\rangle^{(\mathrm{N})}$ and $\left.\left.|\boldsymbol{\mu}\rangle\rangle^{(\mathrm{N})}+\gamma_{k}|\boldsymbol{\Omega}\rangle\right\rangle^{(\mathrm{D})}+\delta_{k}|\boldsymbol{\Omega}\rangle\right\rangle^{(\mathrm{N})}$ with $k=1,2$ and $\alpha_{k}, \beta_{k}, \gamma_{k}, \delta_{k}$ any choice of complex coefficients (such that the resulting four vectors are still linearly independent) also gives four boundary states solving the Cardy condition. Of course, the Cardy condition is only one of many necessary conditions for a consistent boundary theory, and one should therefore not expect that it alone leads to a unique set of boundary states. In the following we shall only consider the ansatz (3.14), and show that it passes a large number of additional consistency checks.

In any case, given any four linearly independent and consistent boundary states, it is clear that one cannot construct a fifth one. For, if there were five linearly independent boundary states, there would be two boundary states that involve $|\boldsymbol{\Omega}\rangle\rangle^{(\mathrm{D})}$ and $\left.|\boldsymbol{\omega}\rangle\right\rangle^{(\mathrm{D})}$, respectively. Their relative overlap then leads to a term proportional to $f_{1}(q)^{2}$ in the closed string channel, which gives rise to $\tilde{\tau} f_{1}(\tilde{q})^{2}$ in the open string; this does not have an interpretation as an open string trace. The fact that there are four boundary states also ties in nicely with the fact that only four of the five chiral torus amplitudes of [51] correspond to characters of highest weight representations. Some aspects of the modular properties of the triplet theory were also studied in [52, 53, 54].

\subsection{One point functions}

It is straightforward to deduce from these boundary states the expressions for the bulk one point functions. In fact, the above boundary states are defined on the disc, and thus we can read off the disc one-point functions from them directly

$$
\begin{aligned}
& \langle\boldsymbol{\Omega}(0)\rangle_{\mathrm{disc}}^{\mathrm{D}, \eta}=\frac{\eta}{\sqrt{4 \pi}} \quad\langle\boldsymbol{\Omega}(0)\rangle_{\mathrm{disc}}^{\mathrm{N}, \eta}=0 \\
& \langle\boldsymbol{\omega}(0)\rangle_{\text {disc }}^{\mathrm{D}, \eta}=0 \quad\langle\boldsymbol{\omega}(0)\rangle_{\mathrm{disc}}^{\mathrm{N}, \eta}=-\eta \sqrt{4 \pi} \\
& \langle\boldsymbol{\mu}(0)\rangle_{\mathrm{disc}}^{\mathrm{D}, \eta}=\frac{1}{2} \quad\langle\boldsymbol{\mu}(0)\rangle_{\mathrm{disc}}^{\mathrm{N}, \eta}=1 .
\end{aligned}
$$

Given the one point functions on the disc, we can apply a conformal transformation to obtain the one-point functions on the upper half plane (uhp). For the $\boldsymbol{\Omega}$ and $\boldsymbol{\mu}$ field this is straightforward, but for $\boldsymbol{\omega}$ we have to bear in mind that it is not an eigenvector of $L_{0}$ (or $\left.\bar{L}_{0}\right)$. In particular, we therefore have for any boundary condition $b$

$$
\begin{aligned}
\langle\boldsymbol{\Omega}(i y)\rangle_{\mathrm{uhp}}^{b} & =\lambda \cdot\langle\boldsymbol{\Omega}(0)\rangle_{\mathrm{disc}}^{b} \\
\langle\boldsymbol{\omega}(i y)\rangle_{\mathrm{uhp}}^{b} & =\lambda \cdot\left(\langle\boldsymbol{\omega}(0)\rangle_{\text {disc }}^{b}-2 \log (2 y)\langle\boldsymbol{\Omega}(0)\rangle_{\text {disc }}^{b}\right) \\
\langle\boldsymbol{\mu}(i y)\rangle_{\mathrm{uhp}}^{b} & =\lambda \cdot(2 y)^{\frac{1}{4}}\langle\boldsymbol{\mu}(0)\rangle_{\text {disc }}^{b},
\end{aligned}
$$

where $\lambda$ is a constant that describes the relative normalisation of the two amplitudes. We 
therefore obtain

$$
\begin{array}{ll}
\langle\boldsymbol{\Omega}(i y)\rangle_{\mathrm{uhp}}^{\mathrm{D}, \eta}=\lambda \cdot \frac{\eta}{\sqrt{4 \pi}} & \langle\boldsymbol{\Omega}(i y)\rangle_{\mathrm{uhp}}^{\mathrm{N}, \eta}=0 \\
\langle\boldsymbol{\omega}(i y)\rangle_{\mathrm{uhp}}^{\mathrm{D}, \eta}=-2 \lambda \cdot \log (2 y) \frac{\eta}{\sqrt{4 \pi}} & \langle\boldsymbol{\omega}(i y)\rangle_{\mathrm{uhp}}^{\mathrm{N}, \eta}=-\lambda \cdot \eta \sqrt{4 \pi} \\
\langle\boldsymbol{\mu}(i y)\rangle_{\mathrm{uhp}}^{\mathrm{D}, \eta}=\lambda \cdot \frac{1}{2}(2 y)^{\frac{1}{4}} & \langle\boldsymbol{\mu}(i y)\rangle_{\mathrm{uhp}}^{\mathrm{N}, \eta}=\lambda \cdot(2 y)^{\frac{1}{4}} .
\end{array}
$$

\section{Operator algebra on the boundary}

The above boundary states determine the triplet representations that appear in the various open string spectra, up to the ambiguity $\chi_{\mathcal{R}_{0}}=\chi_{\mathcal{R}_{1}}=2 \chi_{\mathcal{V}_{0}}+2 \chi_{\mathcal{V}_{1}}$. As already mentioned in section [3.3, we make the ansatz that the space of states on a strip with $(\mathrm{N}, \pm)$-boundary conditions form the representations $\mathcal{R}_{0}$ and $\mathcal{R}_{1}$ as suggested by the notation used in (3.15).

Now we want to check that these boundary operators form indeed an associative algebra. For the case of the $(\mathrm{D}, \pm)$-boundary conditions, the open string spectrum is just the vacuum representation of the triplet algebra which is associative (since the triplet algebra defines a consistent vertex operator algebra). The situation for the $(\mathrm{N}, \pm)$-boundary is however more interesting.

\subsection{The boundary OPE $\omega(x) \omega(0)$}

From the analysis of the cylinder partition functions in section 3.3 we derived the ansatz that the space of boundary fields on the $(\mathrm{N}, \pm)$-boundary forms the representation $\mathcal{R}_{0}$ under the action of the triplet algebra. At level 0 we thus have two linearly independent states, $\omega$ and $\Omega=L_{0} \omega$. The field $\Omega(x)$ will be the identity field on the $(\mathrm{N}, \pm)$-boundary, and according to the discussion in appendix C.4 we choose $\omega$ such that it has the following 2-point function on the upper half plane

$$
\langle\omega(x) \omega(y)\rangle_{\mathrm{uhp}}^{\mathrm{N}, \eta}=-2 \log (x-y)\langle\omega(0)\rangle_{\mathrm{uhp}}^{\mathrm{N}, \eta}, \quad \text { where } \quad \eta= \pm, \quad x>y .
$$

The boundary 1-point function $\langle\omega(0)\rangle_{\text {uhp }}^{\mathrm{N}, \eta}$ (not to be confused with the bulk 1-point function $\langle\boldsymbol{\omega}(i y)\rangle_{\text {uhp }}^{\mathrm{N}, \eta}$ in (3.19) $)$ is independent of the insertion point, and its value will be determined in section 5.1 .3 below.

In order to characterise higher order corrections we need to introduce some notation. In the following $\mathcal{O}_{\log }\left(x^{\ell}\right)$ will denote the functions $f(x)$ for which there is an $n>0$ such that $f(x) /\left(x^{\ell} \cdot \log (x)^{n}\right) \stackrel{x \rightarrow 0}{\longrightarrow}$ const. Furthermore, by $\mathcal{O}_{\log }\left[h, x^{\ell}\right]$ we refer to a state that can be written in the form $\sum_{i} f_{i}(x) v_{i}$ such that each $f_{i}(x)$ is of order $\mathcal{O}_{\log }\left(x^{\ell}\right)$, and each vector $v_{i}$ has (generalised) $L_{0}$-weight greater or equal to $h$.

With these conventions we now make the ansatz for the leading order of the boundary OPE of $\omega$

$$
\omega(x) \omega(0)=f(x) \Omega(0)+g(x) \omega(0)+\mathcal{O}_{\log }[h=1, x] .
$$


The functions $f(x)$ and $g(x)$ can be fixed up to constants by requiring compatibility with the action of $L_{0}$ as follows. Acting with $L_{0}$ on both sides of (4.2) gives

$$
\left(\Omega(x)+x \frac{\partial}{\partial x} \omega(x)\right) \omega(0)+\omega(x) \Omega(0)=g(x) \Omega(0)+\mathcal{O}_{\log }[h=1, x] .
$$

Substituting the ansatz for the OPE and using that $\Omega(x)$ is the identity field results in

$$
\left(x g^{\prime}(x)+2\right) \omega(0)+x f^{\prime}(x) \Omega(0)+\mathcal{O}_{\log }[h=1, x]=g(x) \Omega(0)+\mathcal{O}_{\log }[h=1, x] .
$$

Comparing coefficients gives two first order differential equations for $f$ and $g$ which are solved by $g(x)=-2 \log x+C_{1}$ and $f(x)=-(\log x)^{2}+C_{1} \log x+C_{2}$ for some constants $C_{1}$, $C_{2}$. Applying the OPE to the 2-point function in (4.1) further determines $C_{1}=0$ so that altogether

$$
\omega(x) \omega(0)=\left(C_{\omega}^{\eta}-(\log x)^{2}\right) \Omega(0)-2 \log x \omega(0)+\mathcal{O}_{\log }[h=1, x] .
$$

Here $C_{\omega}^{\eta}$ is a constant that potentially depends on the boundary condition $(\mathrm{N}, \eta)$. To determine this constant one could try to carry out a crossing calculation for the correlator of four $\omega$-fields on the upper half plane. This is done in appendix F.1] and turns out not to pose any restrictions on $C_{\omega}^{\eta}$. We will fix this constant in the next section when looking at boundary changing fields.

\subsection{The boundary OPE $\mu(x) \omega(0)$}

Up to now we have only considered boundary preserving operators. According to (3.15) the space of boundary changing fields that separate a $(\mathrm{N}, \eta)$-boundary condition from a $(\mathrm{D}, \eta)$ boundary condition forms the representation $\mathcal{V}_{-\frac{1}{8}}$ under the action of the triplet algebra. To be specific, let us denote by $\mu^{\eta}(x)$ the boundary changing field that interpolates between the $(\mathrm{N}, \eta)$ boundary condition for boundary points $y<x$, and the $(\mathrm{D}, \eta)$ boundary condition for boundary points $y>x$. We also denote by $\tilde{\mu}^{\eta}(x)$ the boundary changing field which conversely interpolates between $(\mathrm{D}, \eta)$ for $y<x$ and $(\mathrm{N}, \eta)$ for $y>x$. We begin by considering the OPE of $\mu^{\eta}(x)$ with $\omega(0)$ to first subleading order. The representation $\mathcal{V}_{-\frac{1}{8}}$ has one-dimensional eigenspaces for the $L_{0}$ eigenvalues $-\frac{1}{8}$ and $-\frac{1}{8}+1$, spanned by $\mu$ and $L_{-1} \mu$, respectively, so that we can make the ansatz (for $x>0$ )

$$
\mu^{\eta}(x) \omega(0)=f(x) \mu^{\eta}(0)+g(x) L_{-1} \mu^{\eta}(0)+\mathcal{O}_{\log }\left[h=-\frac{1}{8}+2, x^{2}\right],
$$

where $f$ and $g$ are functions. As before, acting with $L_{0}$ on both sides results in a differential equation for $f(x)$, namely this time $x f^{\prime}(x)+1=0$. Acting with $L_{1}$ on both sides gives in addition the condition

$$
\left(-\frac{1}{4} x+x^{2} \frac{\partial}{\partial x}\right)\left(f(x) \mu(0)+g(x) L_{-1} \mu+\cdots\right)=g(x) L_{1} L_{-1} \mu(0)+\mathcal{O}_{\log }\left[h=-\frac{1}{8}+1, x^{2}\right],
$$

which shows that $g(x)=x(f(x)+4)$. Thus altogether

$$
\mu^{\eta}(x) \omega(0)=\left(C_{\mu \omega}^{\eta}-\log x\right) \mu^{\eta}(0)+x\left(C_{\mu \omega}^{\eta}+4-\log x\right) L_{-1} \mu^{\eta}(0)+\mathcal{O}_{\log }\left[h=-\frac{1}{8}+2, x^{2}\right],
$$


for some constant $C_{\mu \omega}^{\eta}$ to be determined below in a crossing calculation.

As another ingredient in the crossing calculation we need two- and three-point functions involving boundary changing fields. Let us write $\tilde{\mu}_{\infty}^{\eta}$ for the boundary field $\tilde{\mu}^{\eta}$ placed at infinity on the upper half plane (see appendix C for a more careful treatment of field insertions at infinity). Then $\left\langle\tilde{\mu}_{\infty}^{\eta} \mu^{\eta}(x)\right\rangle_{\mathrm{uhp}}$ is independent of $x$ and gives the normalisation of the product $\tilde{\mu}^{\eta} \mu^{\eta}$. We will not fix this normalisation, but rather leave the two-point correlator explicitly in intermediate expressions. The three-point function is fixed by Möbius covariance up to constants. One finds

$$
\left\langle\tilde{\mu}_{\infty}^{\eta} \mu^{\eta}(x) \omega(0)\right\rangle_{\mathrm{uhp}}=\left(C_{\mu \omega}^{\eta}-\log x\right)\left\langle\tilde{\mu}_{\infty}^{\eta} \mu^{\eta}(0)\right\rangle_{\mathrm{uhp}}
$$

where the appearance of the OPE coefficient $C_{\mu \omega}^{\eta}$ can be deduced by comparing the small $x$ behaviour of (4.9) with the OPE (4.8).

We have now gathered all ingredients for the crossing calculation. Consider the four point function

$$
F(x)=\left\langle\tilde{\mu}_{\infty}^{\eta} \mu^{\eta}(1) \omega(x) \omega(0)\right\rangle_{\mathrm{uhp}}, \quad 0<x<1 .
$$

The asymptotic expansions as given by the OPEs (4.5) and (4.8) are

$$
F(\varepsilon) \underset{\varepsilon \rightarrow 0}{=}\left(C_{\omega}^{\eta}-2 C_{\mu \omega}^{\eta} \log \varepsilon-(\log \varepsilon)^{2}+\mathcal{O}_{\log }(\varepsilon)\right) \cdot\left\langle\tilde{\mu}_{\infty}^{\eta} \mu^{\eta}(0)\right\rangle_{\mathrm{uhp}}
$$

and

$$
F(1-\varepsilon) \underset{\varepsilon \rightarrow 0}{=}\left(\left(C_{\mu \omega}^{\eta}\right)^{2}-C_{\mu \omega}^{\eta} \log \varepsilon-4 \varepsilon+\mathcal{O}_{\log }\left(\varepsilon^{2}\right)\right) \cdot\left\langle\tilde{\mu}_{\infty}^{\eta} \mu^{\eta}(0)\right\rangle_{\mathrm{uhp}} .
$$

On the other hand, using a null vector argument outlined in appendix E.3, $F(x)$ has to be equal to

$$
F(x)=-C_{1} h(x)^{2}-C_{2} \log (1-x)+C_{4} h(x)+C_{5}, \quad h(x)=\log \left(\frac{1-\sqrt{1-x}}{1+\sqrt{1-x}}\right)
$$

for an appropriate choice of constants $C_{1}, C_{2}, C_{4}$ and $C_{5}$. Using the asymptotic behaviour (4.12) to fix these constants results in

$$
\begin{array}{ll}
C_{1}=\left\langle\tilde{\mu}_{\infty}^{\eta} \mu^{\eta}(0)\right\rangle_{\mathrm{uhp}}, & C_{2}=C_{\mu \omega}^{\eta} \cdot\left\langle\tilde{\mu}_{\infty}^{\eta} \mu^{\eta}(0)\right\rangle_{\mathrm{uhp}} \\
C_{4}=0, & C_{5}=\left(C_{\mu \omega}^{\eta}\right)^{2} \cdot\left\langle\tilde{\mu}_{\infty}^{\eta} \mu^{\eta}(0)\right\rangle_{\mathrm{uhp}} .
\end{array}
$$

One can now compute the small $x$ behaviour of $F(x)$ to be

$$
F(x)=\left(\left(C_{\mu \omega}^{\eta}\right)^{2}-(2 \log 2)^{2}+4 \log 2 \cdot \log x-(\log x)^{2}+\mathcal{O}_{\log }(x)\right)\left\langle\tilde{\mu}_{\infty}^{\eta} \mu^{\eta}(0)\right\rangle_{\mathrm{uhp}} .
$$

Comparing to the asymptotic behaviour (4.11) obtained using the OPE directly shows that there is a unique consistent choice for the boundary OPE coefficients involved, namely

$$
C_{\omega}^{\eta}=0, \quad C_{\mu \omega}^{\eta}=-2 \log 2 .
$$


This confirms the operator product expansion (2.23) quoted in the summary section. It also shows that (at least to this order) the boundary fields define a consistent (associative) operator product. 4

It should be pointed out that while the above arguments fix the leading order in the operator product expansion (4.5) of two $\omega$ fields, in a more detailed analysis one finds that there are still two undetermined constants at subleading orders. This is discussed in appendix F.2.

\section{Bulk-boundary OPE and factorisation}

Next we want to determine the bulk-boundary operator product expansion of the bulk fields $\boldsymbol{\omega}$ and $\boldsymbol{\mu}$ near a $(\mathrm{D}, \pm)$ and $(\mathrm{N}, \pm)$-boundary. This will allow us to check some of the factorisation constraints, in particular the compatibility of our boundary conditions with the bulk operator product expansion.

\subsection{Bulk-boundary OPE}

Let $\phi(i y)$ be a Virasoro primary bulk field in the upper half plane, with $y>0$ the distance to the boundary. Depending on whether we put the $(\mathrm{D}, \pm)$ or $(\mathrm{N}, \pm)$-boundary condition on the real line, we can make the following ansatz for the leading order in the bulk-boundary operator product expansion,

$$
\begin{aligned}
& \left.\phi(i y)\right|_{\mathrm{D}, \pm}=a(y) \Omega(0)+\mathcal{O}_{\log }\left[h=2, y^{2}\right], \\
& \left.\phi(i y)\right|_{\mathrm{N}, \pm}=b(y) \Omega(0)+c(y) \omega(0)+\mathcal{O}_{\log }[h=1, y],
\end{aligned}
$$

with $\mathcal{O}_{\log }[h, y]$ as defined in section 4.1. Here $a(y), b(y), c(y)$ are functions that depend on the bulk field $\phi$ and the boundary condition. Note that the operator product expansion for the $(\mathrm{D}, \pm)$-boundary condition does not contain a linear $y$-term since the boundary fields transform in the representation $\mathcal{V}_{0}$ that does not have a state at conformal weight one.

To determine the functions $a(y), b(y), c(y)$, one first demands consistency with the action of the Virasoro zero mode on the boundary, $L_{0}^{\text {bnd }}$. Recall from [55] that the Virasoro modes acting on boundary fields on the upper half plane are build by integrating $T(z)$ and $\bar{T}(z)$ on half-circles on the upper half plane. Correspondingly, the commutation relation with the bulk-field $\phi(z)$ now reads

$$
\left[L_{m}^{\mathrm{bnd}}, \boldsymbol{\phi}(z)\right]=\left(\left((m+1) z^{m} L_{0}+z^{m+1} L_{-1}+(m+1)\left(z^{*}\right)^{m} \bar{L}_{0}+\left(z^{*}\right)^{m+1} \bar{L}_{-1}\right) \boldsymbol{\phi}\right)(z) .
$$

\footnotetext{
${ }^{4}$ After a short calculation using (4.1), (4.9) and (4.16) one finds for the boundary changing fields the two OPEs $\mu^{\eta}(x) \tilde{\mu}^{\eta}(0)=N_{1} x^{\frac{1}{4}}(\Omega(0)+\ldots)$ and $\tilde{\mu}^{\eta}(x) \mu^{\eta}(0)=N_{2} x^{\frac{1}{4}}(\omega(0)+\log (x / 4) \Omega(0)+\ldots)$, where $x>0$ and $N_{1}=\left\langle\mu_{\infty}^{\eta} \tilde{\mu}^{\eta}(0)\right\rangle_{\mathrm{uhp}} /\langle\mathbf{1}\rangle_{\mathrm{uhp}}^{\mathrm{D}, \eta}$ and $N_{2}=\left\langle\tilde{\mu}_{\infty}^{\eta} \mu^{\eta}(0)\right\rangle_{\mathrm{uhp}} /\langle\omega(0)\rangle_{\mathrm{uhp}}^{\mathrm{N}, \eta}$ are constants related to the normalisation of the fields, see also section 5.1 .3 below. In the first case, a stretch of $(\mathrm{N}, \eta)$ boundary is collapsed and one is left with boundary fields on $(\mathrm{D}, \eta)$; in the second case a stretch of $(\mathrm{D}, \eta)$ boundary disappears and the resulting boundary fields live on the $(\mathrm{N}, \eta)$ boundary. However, we will not need these OPEs in the present paper.
} 
In the case $z=i y$ and $m=0$ this simplifies to

$$
\left[L_{0}^{\mathrm{bnd}}, \boldsymbol{\phi}(i y)\right]=\left(\left(L_{0}+\bar{L}_{0}\right) \boldsymbol{\phi}\right)(i y)+y \frac{\partial}{\partial y} \phi(i y) .
$$

Just as for the OPEs (4.2) and (4.6), by acting with $L_{0}^{\text {bnd }}$ on both sides of (5.1) we obtain differential equations for $a(y), b(y), c(y)$. For the bulk fields $\boldsymbol{\omega}$ and $\boldsymbol{\mu}$ one obtains in this way

$$
\begin{aligned}
\left.\boldsymbol{\omega}(i y)\right|_{\mathrm{D}, \eta} & =\left(C_{\omega}^{\mathrm{D}, \eta}-2 \log y\right) \Omega(0)+\mathcal{O}_{\log }\left[h=2, y^{2}\right] \\
\left.\boldsymbol{\omega}(i y)\right|_{\mathrm{N}, \eta} & =D_{\omega}^{\mathrm{N}, \eta} \omega(0)+\left(\left(D_{\omega}^{\mathrm{N}, \eta}-2\right) \log y+C_{\omega}^{\mathrm{N}, \eta}\right) \Omega(0)+\mathcal{O}_{\log }[h=1, y] \\
\left.\boldsymbol{\mu}(i y)\right|_{\mathrm{D}, \eta} & =C_{\mu}^{\mathrm{D}, \eta}(2 y)^{\frac{1}{4}}\left(\Omega(0)+\mathcal{O}_{\log }\left[h=2, y^{2}\right]\right) \\
\left.\boldsymbol{\mu}(i y)\right|_{\mathrm{N}, \eta} & =D_{\mu}^{\mathrm{N}, \eta}(2 y)^{\frac{1}{4}}\left(\omega(0)+\left(\log y+C_{\mu}^{\mathrm{N}, \eta}\right) \Omega(0)+\mathcal{O}_{\log }[h=1, y]\right)
\end{aligned}
$$

with a number of undetermined constants. Some of these are fixed by comparing the OPEs to the one-point functions on the upper half plane (3.19). For example, using the first OPE in (5.4) we get

$$
\langle\boldsymbol{\omega}(i y)\rangle_{\mathrm{uhp}}^{\mathrm{D}, \eta}=\left(C_{\omega}^{\mathrm{D}, \eta}-2 \log y\right)\langle\mathbf{1}\rangle_{\mathrm{uhp}}^{\mathrm{D}, \eta}+\mathcal{O}_{\log }\left(y^{2}\right)=-2 \log (2 y)\langle\mathbf{1}\rangle_{\mathrm{uhp}}^{\mathrm{D}, \eta},
$$

where in the last equality the exact expression from (3.19) was substituted. Here $\langle\mathbf{1}\rangle$ denotes the correlator with no field insertions. Note that an insertion of the bulk vacuum $\Omega(z)$ or the boundary vacuum $\Omega(x)$ counts as no field insertion. In this way we arrive at

$$
C_{\omega}^{\mathrm{D}, \eta}=-2 \log 2, \quad C_{\mu}^{\mathrm{D}, \eta}=\eta \sqrt{\pi} .
$$

This determines the bulk-boundary OPE of $\boldsymbol{\omega}$ and $\boldsymbol{\mu}$ close to a $(\mathrm{D}, \eta)$-boundary. To find the coefficients for a $(\mathrm{N}, \eta)$-boundary we perform a crossing calculation in the presence of a boundary changing field.

Consider a bulk field $\phi(z)$ on the upper half plane, where on the positive real axis we have the boundary condition $(\mathrm{D}, \eta)$, and on the negative real axis the boundary condition $(\mathrm{N}, \eta)$. At the points 0 and $\infty$ boundary changing fields have to be inserted, and the correlator we will consider is

$$
\left\langle\tilde{\mu}_{\infty}^{\eta} \mu^{\eta}(0) \phi\left(e^{i \theta}\right)\right\rangle_{\mathrm{uhp}}
$$

The asymptotics of this block in the $\theta \rightarrow 0$ limit is known from the OPE (5.4), together with (5.6). Matching this with the four-point blocks in appendix E allows us to determine (5.7) uniquely. Finally, evaluating the behaviour for $\theta \rightarrow \pi$ fixes the remaining constants in (5.4).

To carry out this calculation, we need to relate the correlator (5.7) to the four-point blocks with insertions at $\infty, 1, x$ and 0 in appendix E. Suppose the bulk field $\phi$ is of the form $\phi=\phi_{1} \otimes \phi_{2}+\mathcal{N}$. Then the correlator (5.7) is equal to a conformal block with insertions $\left\langle\mu \mid \mu(1) \phi_{1}\left(e^{i \theta}\right) \phi_{2}\left(e^{-i \theta}\right)\right\rangle$. The relevant Möbius transformation is $\zeta \mapsto 1-e^{i \theta} \zeta$, so that the correlator (5.7) is an element of the following space of blocks,

$$
\left\langle\tilde{\mu}_{\infty}^{\eta} \mu^{\eta}(0) \phi\left(e^{i \theta}\right)\right\rangle_{\mathrm{uhp}} \in\left\{\left\langle\mu \mid \mu(1) \tilde{\phi}_{1}(x) \tilde{\phi}_{2}(0)\right\rangle\right\}, \quad x=1-e^{2 i \theta},
$$

where $\tilde{\phi}_{1}=e^{i(\theta+\pi) L_{0}} \phi_{1}$ and $\tilde{\phi}_{2}=e^{i(\theta+\pi) L_{0}} \phi_{2}$. Let us first consider the case $\boldsymbol{\phi}=\boldsymbol{\mu}$ and then go on to $\phi=\omega$. 


\subsubsection{The bulk field $\mu$ in the presence of boundary changing fields}

Substituting the general solution for the four-point block with four $\mu$-insertions from (E.10) into (5.8) gives

$$
\left\langle\tilde{\mu}_{\infty}^{\eta} \mu^{\eta}(0) \boldsymbol{\mu}\left(e^{i \theta}\right)\right\rangle_{\mathrm{uhp}}=(2 \sin \theta)^{\frac{1}{4}} e^{i \theta / 2}\left(C_{1} F(x)+C_{2} G(x)\right) \quad \text { for some } C_{1}, C_{2} \in \mathbb{C},
$$

where as before $x=1-e^{2 i \theta}$ and furthermore

$$
\begin{aligned}
& F(x)={ }_{2} F_{1}\left(\frac{1}{2}, \frac{1}{2} ; 1 ; x\right)=1+\frac{1}{4} x+\mathcal{O}\left(x^{2}\right), \\
& G(x)={ }_{2} F_{1}\left(\frac{1}{2}, \frac{1}{2} ; 1 ; 1-x\right)=\frac{1}{\pi}\left(4 \log 2-\log x+\left(\log 2-\frac{1}{2}-\frac{1}{4} \log x\right) x+\mathcal{O}_{\log }\left(x^{2}\right)\right) .
\end{aligned}
$$

Using the OPE (5.4) close to a (D, $)$-boundary gives the asymptotic behaviour

$$
\left\langle\tilde{\mu}_{\infty}^{\eta} \mu^{\eta}(0) \boldsymbol{\mu}\left(e^{i \theta}\right)\right\rangle_{\mathrm{uhp}}=\eta \sqrt{\pi}(2 \theta)^{\frac{1}{4}}\left(\left\langle\tilde{\mu}_{\infty}^{\eta} \mu^{\eta}(0)\right\rangle_{\mathrm{uhp}}+\mathcal{O}_{\log }\left(\theta^{2}\right)\right)
$$

This fixes $C_{1}=\eta \sqrt{\pi}\left\langle\tilde{\mu}_{\infty}^{\eta} \mu^{\eta}(0)\right\rangle_{\text {uhp }}$ and $C_{2}=0$, so that altogether the correlator is equal to

$$
\left\langle\tilde{\mu}_{\infty}^{\eta} \mu^{\eta}(0) \boldsymbol{\mu}\left(e^{i \theta}\right)\right\rangle_{\mathrm{uhp}}=\eta \sqrt{\pi}(2 \sin \theta)^{\frac{1}{4}} e^{i \theta / 2}{ }_{2} F_{1}\left(\frac{1}{2}, \frac{1}{2} ; 1 ; 1-e^{2 i \theta}\right)\left\langle\tilde{\mu}_{\infty}^{\eta} \mu^{\eta}(0)\right\rangle_{\mathrm{uhp}} .
$$

Next we need to evaluate the asymptotic behaviour for $\theta \rightarrow \pi$. To do so, first note that varying the angle $\theta$ from 0 to $\pi$ amounts to moving the point $x=1-e^{2 i \theta}$ counter-clockwise around the point 1 . Since 1 is a branchpoint of $F(x)$, we have to analytically continue $F(x)$ before evaluating the $x \rightarrow 0$ behaviour (which amounts to $\theta \rightarrow \pi$ ). Let $\mathcal{C}_{1}$ be the analytic continuation of the argument $x$ counter-clockwise around the point 1 . For the corresponding monodromy one finds

$$
F(x) \stackrel{\mathcal{C}_{1}}{\longrightarrow} F(x)-2 i G(x), \quad G(x) \stackrel{\mathcal{C}_{1}}{\longrightarrow} G(x) .
$$

The asymptotics of (5.12) is then given by

$$
\begin{aligned}
& \left\langle\tilde{\mu}_{\infty}^{\eta} \mu^{\eta}(0) \boldsymbol{\mu}\left(e^{i(\pi-\varepsilon)}\right)\right\rangle_{\mathrm{uhp}} \\
& =\eta \sqrt{\pi}(2 \sin \varepsilon)^{\frac{1}{4}} i e^{-i \varepsilon / 2}\left(F\left(1-e^{-2 i \varepsilon}\right)-2 i G\left(1-e^{-2 i \varepsilon}\right)\right)\left\langle\tilde{\mu}_{\infty}^{\eta} \mu^{\eta}(0)\right\rangle_{\mathrm{uhp}} \\
& =\eta \sqrt{\pi}(2 \varepsilon)^{\frac{1}{4}}\left(\frac{6}{\pi} \log 2-\frac{2}{\pi} \log \varepsilon+\mathcal{O}_{\log }(\varepsilon)\right)\left\langle\tilde{\mu}_{\infty}^{\eta} \mu^{\eta}(0)\right\rangle_{\mathrm{uhp}} .
\end{aligned}
$$

This has to be compared with the asymptotics obtained using the OPE (5.4) of $\boldsymbol{\mu}$ close to a $(\mathrm{N}, \eta)$-boundary, which is

$$
\left\langle\tilde{\mu}_{\infty}^{\eta} \mu^{\eta}(0) \boldsymbol{\mu}\left(e^{i(\pi-\varepsilon)}\right)\right\rangle_{\mathrm{uhp}}=D_{\mu}^{\mathrm{N}, \eta}(2 \varepsilon)^{\frac{1}{4}}\left(C_{\mu}^{\mathrm{N}, \eta}-2 \log 2+\log \varepsilon+\mathcal{O}_{\log }(\varepsilon)\right)\left\langle\tilde{\mu}_{\infty}^{\eta} \mu^{\eta}(0)\right\rangle_{\mathrm{uhp}}
$$

where we also used the boundary three point function (4.9) and the structure constant (4.16). The resulting values of the so far undetermined constants are now fixed uniquely to be

$$
D_{\mu}^{\mathrm{N}, \eta}=-2 \eta \pi^{-\frac{1}{2}}, \quad C_{\mu}^{\mathrm{N}, \eta}=-\log 2 .
$$

Altogether, for $\boldsymbol{\mu}$ we obtain the bulk-boundary OPE quoted in (2.24) in the introduction. 


\subsubsection{The bulk field $\omega$ in the presence of boundary changing fields}

For $\phi=\boldsymbol{\omega}=\omega \otimes \omega+\mathcal{N}$ we need the space of blocks (5.8) with $\tilde{\phi}_{1}=\tilde{\phi}_{2}=\omega+i(\theta+\pi) \Omega$. The relevant individual four-point blocks are given in (E.14) and (E.16). This results in the following ansatz for the correlator

$$
\begin{aligned}
\left\langle\tilde{\mu}_{\infty}^{\eta} \mu^{\eta}(0) \boldsymbol{\omega}\left(e^{i \theta}\right)\right\rangle_{\mathrm{uhp}} & \\
=- & C_{1} h(x)^{2}+C_{4} h(x)+C_{1} \theta^{2}+i\left(C_{3}-C_{2}\right) \theta+C_{5}+i \pi\left(C_{2}+C_{3}\right)-\pi^{2} C_{1} \\
=- & \left(\pi^{2}+\left(\frac{i \pi}{2}+\log 2\right)^{2}\right) C_{1}+i \pi\left(C_{3}+C_{2}\right)-\left(\frac{i \pi}{2}+\log 2\right) C_{4}+C_{5} \\
& \quad+\left(C_{4}+2\left(\frac{i \pi}{2}+\log 2\right) C_{1}\right) \log \theta-C_{1}(\log \theta)^{2}+i\left(C_{3}-C_{2}\right) \theta+\mathcal{O}_{\log }\left(\theta^{2}\right) .
\end{aligned}
$$

Here $C_{1}, \ldots, C_{5} \in \mathbb{C}$ are constants (used in the same way as in (E.14) and (E.16)), the function $h(x)$ is given in (E.16), and as before $x=1-e^{2 i \theta}$. Using the OPE of $\boldsymbol{\omega}$ close to a $(\mathrm{D}, \eta)$-boundary we obtain

$$
\left\langle\tilde{\mu}_{\infty}^{\eta} \mu^{\eta}(0) \boldsymbol{\omega}\left(e^{i \theta}\right)\right\rangle_{\mathrm{uhp}}=-2 \log (2 \theta)\left\langle\tilde{\mu}_{\infty}^{\eta} \mu^{\eta}(0)\right\rangle_{\mathrm{uhp}}+\mathcal{O}_{\log }\left(\theta^{2}\right) .
$$

Comparing to (5.17) it follows that $C_{1}=0, C_{2}-C_{3}=0$, etc. Altogether, the correlator is simply

$$
\left\langle\tilde{\mu}_{\infty}^{\eta} \mu^{\eta}(0) \boldsymbol{\omega}\left(e^{i \theta}\right)\right\rangle_{\mathrm{uhp}}=-2 \log \left(4 \tan \frac{\theta}{2}\right) \cdot\left\langle\tilde{\mu}_{\infty}^{\eta} \mu^{\eta}(0)\right\rangle_{\mathrm{uhp}} .
$$

For $\theta=\pi-\varepsilon$ and $\varepsilon \rightarrow 0$ this correlator behaves as $\left(-6 \log 2+2 \log \varepsilon+\mathcal{O}_{\log }(\varepsilon)\right)\left\langle\tilde{\mu}_{\infty}^{\eta} \mu^{\eta}(0)\right\rangle_{\text {uhp }}$, which has to be matched against the expression obtained using the OPE close to the (N, $\eta$ ) boundary,

$$
\begin{aligned}
& \left\langle\tilde{\mu}_{\infty}^{\eta} \mu^{\eta}(0) \boldsymbol{\omega}\left(e^{i(\pi-\varepsilon)}\right)\right\rangle_{\mathrm{uhp}} \\
& =\left(-2 \log 2 \cdot D_{\omega}^{\mathrm{N}, \eta}+C_{\omega}^{\mathrm{N}, \eta}+\left(D_{\omega}^{\mathrm{N}, \eta}-2\right) \log \varepsilon+\mathcal{O}_{\log }(\varepsilon)\right)\left\langle\tilde{\mu}_{\infty}^{\eta} \mu^{\eta}(0)\right\rangle_{\mathrm{uhp}},
\end{aligned}
$$

where as before we also used (4.9) and (4.16). The resulting constants are

$$
D_{\omega}^{\mathrm{N}, \eta}=4, \quad C_{\omega}^{\mathrm{N}, \eta}=2 \log 2,
$$

which when inserted into (5.4) indeed gives (2.24).

\subsubsection{Bulk one-point correlators on the upper half plane}

Having established the list of OPEs in (2.24), we note that $\langle\boldsymbol{\omega}(i y)\rangle_{\text {uhp }}^{\mathrm{N}, \eta}=4\langle\omega(0)\rangle_{\text {uhp }}^{\mathrm{N}, \eta}$. Together with (3.19) we therefore have

$$
\langle\mathbf{1}\rangle_{\mathrm{uhp}}^{\mathrm{D}, \eta}=\frac{1}{2} \lambda \eta \pi^{-\frac{1}{2}} \quad \text { and } \quad\langle\omega(0)\rangle_{\mathrm{uhp}}^{\mathrm{N}, \eta}=-\frac{1}{2} \lambda \eta \pi^{\frac{1}{2}} .
$$

These are the simplest non-zero correlators on the upper half plane with $(\mathrm{D}, \eta)$ and $(\mathrm{N}, \eta)$ boundary condition, respectively, and we will normalise other uhp-correlators relative to these. For example, for the bulk one-point functions in (3.19) we get

$$
\begin{array}{rlrl}
\langle\boldsymbol{\omega}(i y)\rangle_{\text {uhp }}^{\mathrm{D}, \eta}=-2 \log (2 y)\langle\mathbf{1}\rangle_{\text {uhp }}^{\mathrm{D}, \eta}, & & \langle\boldsymbol{\omega}(i y)\rangle_{\text {uhp }}^{\mathrm{N}, \eta}=4\langle\omega(0)\rangle_{\text {uhp }}^{\mathrm{N}, \eta}, \\
\langle\boldsymbol{\mu}(i y)\rangle_{\text {uhp }}^{\mathrm{D}, \eta}=\eta \pi^{\frac{1}{2}}(2 y)^{\frac{1}{4}}\langle\mathbf{1}\rangle_{\text {uhp }}^{\mathrm{D}, \eta}, & \langle\boldsymbol{\mu}(i y)\rangle_{\text {uhp }}^{\mathrm{N}, \eta}=-2 \eta \pi^{-\frac{1}{2}}(2 y)^{\frac{1}{4}}\langle\omega(0)\rangle_{\text {uhp }}^{\mathrm{N}, \eta} .
\end{array}
$$




\subsection{Factorisation for two bulk fields on the upper half plane}

The computation of the bulk-boundary OPE (2.24) did use implicitly the bulk spectrum of the triplet theory, which entered the process of finding a consistent ansatz for the boundary states. However, we should stress that the bulk OPEs (2.16) have not been used at any point of the calculation so far. These bulk OPEs were determined in [43] by demanding crossing symmetry for correlators on the complex plane without reference to any boundary conditions.

By performing a crossing calculation with the correlator of two bulk fields on the upper half plane, which links the limit where the two bulk fields approach the boundary to the limit where the two bulk fields approach each other, we can compare the bulk OPE to the bulk-boundary OPE coefficients. This provides a significant consistency check (which is sometimes called the cluster condition or classifying algebra) on the bulk-boundary OPE and hence also on the boundary field content found in the boundary state analysis. In [56] the opposite procedure was used to deduce the bulk-boundary OPE from the known bulk OPE.

Consider thus the correlator of two bulk fields $\boldsymbol{\phi}=\phi_{1} \otimes \phi_{2}+\mathcal{N}$ and $\boldsymbol{\psi}=\psi_{1} \otimes \psi_{2}+\mathcal{N}$ on the upper half plane with boundary condition $b$. The chiral Ward identities demand this correlator to be an element of the following space of conformal four-point blocks ${ }^{5}$

$$
\langle\phi(x+i y) \boldsymbol{\psi}(i y)\rangle_{\text {uhp }}^{b} \in\left\{\left\langle\phi_{1}(x+i y) \phi_{2}(x-i y) \psi_{1}(i y) \psi_{2}(-i y)\right\rangle\right\},
$$

where $x, y \in \mathbb{R}_{>0}$. To make use of the four-point blocks derived in appendix $\mathbb{E}$ one has to apply a Möbius transformation to move the insertion points to $\infty, 1, r$ and 0 . We will use

$$
M(\zeta)=\frac{2 i y}{2 i y-x} \cdot \frac{\zeta-i y}{\zeta-(x+i y)},
$$

which takes $x+i y$ to infinity, etc. The scaling factors arising in this transformation are easiest to determine by explicitly working with the local coordinates around the insertion points as in appendix C One finds that the uhp-correlator is equally an element of the following space of blocks,

$$
\langle\boldsymbol{\phi}(x+i y) \boldsymbol{\psi}(i y)\rangle_{\mathrm{uhp}}^{b} \in\left\{\left\langle\tilde{\phi}_{1} \mid \tilde{\phi}_{2}(1) \tilde{\psi}_{2}(r) \tilde{\psi}_{1}(0)\right\rangle\right\}, \quad \text { where } \quad r=\frac{(2 y)^{2}}{x^{2}+(2 y)^{2}}
$$

and

$$
\begin{array}{llll}
\tilde{\phi}_{1}=\left(f_{\infty}\right)^{L_{0}} \phi_{1}, & \tilde{\phi}_{2}=\left(f_{1}\right)^{L_{0}} \phi_{2}, & \tilde{\psi}_{2}=\left(f_{r}\right)^{L_{0}} \psi_{2}, & \tilde{\psi}_{1}=\left(f_{0}\right)^{L_{0}} \psi_{1}, \\
f_{\infty}=\frac{x-2 i y}{2 i x y}, & f_{1}=\frac{x}{2 i y(x-2 i y)}, & f_{r}=\frac{2 i x y}{(x-2 i y)(x+2 i y)^{2}}, & f_{0}=\frac{2 i y}{x(x-2 i y)} .
\end{array}
$$

We will present the calculations for the three correlators $\langle\boldsymbol{\mu}(z) \boldsymbol{\mu}(w)\rangle_{\mathrm{uhp}},\langle\boldsymbol{\mu}(z) \boldsymbol{\omega}(w)\rangle_{\mathrm{uhp}}$, and $\langle\boldsymbol{\omega}(z) \boldsymbol{\omega}(w)\rangle_{\mathrm{uhp}}$, in this order.

\footnotetext{
${ }^{5}$ This is what is sometimes referred to as the doubling trick.
} 


\subsubsection{Two $\mu$ fields on the upper half plane}

In this case one has to apply (5.26) to the case $\phi=\psi=\mu \otimes \mu+\mathcal{N}$. Substituting also the general form (E.10) of the four-point blocks, a short calculation yields

$$
\begin{gathered}
\langle\boldsymbol{\mu}(x+i y) \boldsymbol{\mu}(i y)\rangle_{\mathrm{uhp}}^{b}=\left(\frac{(2 x y)^{2}}{x^{2}+(2 y)^{2}}\right)^{\frac{1}{4}}\left(A_{1} F(r)+A_{2} G(r)\right) \\
\stackrel{y \rightarrow 0}{=}(2 y)^{\frac{1}{2}}\left(A_{1}+A_{2} \frac{2}{\pi} \log 2+A_{2} \frac{2}{\pi} \log \frac{x}{y}+\mathcal{O}_{\log }\left(y^{2}\right)\right) \\
\stackrel{x \rightarrow 0}{=} x^{\frac{1}{2}}\left(A_{1} \frac{6}{\pi} \log 2+A_{2}-A_{1} \frac{2}{\pi} \log \frac{x}{y}+\mathcal{O}_{\log }\left(x^{2}\right)\right)
\end{gathered}
$$

with $F$ and $G$ as in (5.10) and $A_{1}, A_{2} \in \mathbb{C}$ constants to be determined. The $y \rightarrow 0$ behaviour is fixed by the bulk-boundary OPE (2.24),

$$
\langle\boldsymbol{\mu}(x+i y) \boldsymbol{\mu}(i y)\rangle_{\mathrm{uhp}}^{b}=(2 y)^{\frac{1}{2}} \cdot \begin{cases}\pi\langle\mathbf{1}\rangle_{\mathrm{uhp}}^{\mathrm{D}, \eta}+\mathcal{O}_{\log }\left(y^{2}\right) & ; b=\mathrm{D}, \eta \\ -\frac{8}{\pi}\left(\log 2+\log \frac{x}{y}\right)\langle\omega(0)\rangle_{\mathrm{uhp}}^{\mathrm{N}, \eta}+\mathcal{O}_{\log }\left(y^{2}\right) & ; b=\mathrm{N}, \eta\end{cases}
$$

For the (N, $\eta)$-boundary we also used (4.1) and the fact that $\langle\mathbf{1}\rangle_{\text {uhp }}^{\mathrm{N}, \eta}=0$ (cf. (3.19)) and that $\left\langle\omega(x)\left(W_{-1}^{a} \omega\right)(y)\right\rangle_{\text {uhp }}^{\mathrm{N}, \eta}=0$. Comparing to (5.28) yields

$$
\begin{array}{lll}
b=\mathrm{D}, \eta \quad: & A_{1}=\pi\langle\mathbf{1}\rangle_{\text {uhp }}^{\mathrm{D}, \eta}, & A_{2}=0, \\
b=\mathrm{N}, \eta \quad: & A_{1}=0, & A_{2}=-4\langle\omega(0)\rangle_{\text {uhp }}^{\mathrm{N}, \eta} .
\end{array}
$$

By substituting these results into (5.28) we can then evaluate the $x \rightarrow 0$ behaviour.

Alternatively, this behaviour can be obtained using the bulk OPE (2.16). Together with (5.23) one obtains

$$
\langle\boldsymbol{\mu}(x+i y) \boldsymbol{\mu}(i y)\rangle_{\mathrm{uhp}}^{b}=x^{\frac{1}{2}} \cdot \begin{cases}\left(6 \log 2-2 \log \frac{x}{y}\right)\langle\mathbf{1}\rangle_{\mathrm{uhp}}^{\mathrm{D}, \eta}+\mathcal{O}_{\log }\left(x^{2}\right) & ; b=\mathrm{D}, \eta \\ -4\langle\omega(0)\rangle_{\mathrm{uhp}}^{\mathrm{N}, \eta}+\mathcal{O}_{\log }\left(x^{2}\right) & ; b=\mathrm{N}, \eta\end{cases}
$$

in perfect agreement with (5.28) and (5.30).

\subsubsection{One $\mu$ and one $\omega$ field on the upper half plane}

For this calculation we will set $\phi_{1}=\phi_{2}=\mu$ and allow $\psi_{1}, \psi_{2} \in\{\omega, \Omega\}$. Let us abbreviate a conformal four-point block with two $\mu$-insertions and two arbitrary insertions $\alpha, \beta$ as

$$
U(\alpha, \beta)=\langle\mu \mid \mu(1) \alpha(r) \beta(0)\rangle .
$$

Combining (5.26) and (5.27) results in the following expressions for the bulk two-point function on the upper half plane,

$$
\begin{aligned}
\langle\boldsymbol{\mu}(x+i y) \boldsymbol{\psi}(i y)\rangle_{\mathrm{uhp}}^{b}= & (2 y)^{\frac{1}{4}}\left(U\left(\psi_{2}, \psi_{1}\right)+\log f_{r} \cdot U\left(L_{0} \psi_{2}, \psi_{1}\right)\right. \\
& \left.+\log f_{0} \cdot U\left(\psi_{2}, L_{0} \psi_{1}\right)+\log f_{r} \cdot \log f_{0} \cdot U\left(L_{0} \psi_{2}, L_{0} \psi_{1}\right)\right) .
\end{aligned}
$$


The relevant conformal blocks are given in (E.12), (E.14) and (E.16) in terms of five free parameters $C_{1}, \ldots, C_{5}$.

To fix some of these parameters, let us first choose $\psi_{1}=\psi_{2}=\Omega$, i.e. set $\boldsymbol{\psi}=N=$ $\Omega \otimes \Omega+\mathcal{N}$. Since $\Omega \otimes \Omega \in \mathcal{N}$, we have $N=0$ in the quotient space $\mathcal{H}^{\text {bulk }}$, and a correlator involving an insertion of $N$ has to vanish. In particular,

$$
0=\langle\boldsymbol{\mu}(x+i y) N(i y)\rangle_{\mathrm{uhp}}^{b}=(2 y)^{\frac{1}{4}} U(\Omega, \Omega)=(2 y)^{\frac{1}{4}} C_{1}
$$

which implies that $C_{1}=0$, independent of the choice of boundary condition $b$. Similarly, we have $\omega \otimes \Omega-\Omega \otimes \omega \in \mathcal{N}$. If we set $V_{l}=\omega \otimes \Omega+\mathcal{N}$ and $V_{r}=\Omega \otimes \omega+\mathcal{N}$, then $V_{l}=V_{r}$ in $\mathcal{H}^{\text {bulk }}$ and hence

$$
\begin{aligned}
0= & \left\langle\boldsymbol{\mu}(x+i y) V_{l}(i y)\right\rangle_{\mathrm{uhp}}^{b}-\left\langle\boldsymbol{\mu}(x+i y) V_{r}(i y)\right\rangle_{\mathrm{uhp}}^{b} \\
& =(2 y)^{\frac{1}{4}}(U(\Omega, \omega)-U(\omega, \Omega))=(2 y)^{\frac{1}{4}}\left(C_{2}-C_{3}\right),
\end{aligned}
$$

where in the last step we used that $C_{1}=0$. We thus get the additional condition $C_{2}=C_{3}$. Altogether, the functional form of the two-point function of $\boldsymbol{\mu}$ and $\boldsymbol{\omega}$ is now restricted to

$$
\begin{aligned}
& \langle\boldsymbol{\mu}(x+i y) \boldsymbol{\omega}(i y)\rangle_{\mathrm{uhp}}^{b}=(2 y)^{\frac{1}{4}}\left(C_{2}(\pi i+\log r-2 \log x)+C_{4} h(r)+C_{5}\right) \\
& \stackrel{y \rightarrow 0}{=}(2 y)^{\frac{1}{4}}\left(C_{2}(i \pi+2 \log 2)+C_{5}-2\left(2 C_{2}+C_{4}\right) \log x+2\left(C_{2}+C_{4}\right) \log y+\mathcal{O}_{\log }\left(y^{2}\right)\right) \\
& \stackrel{x \rightarrow 0}{=}(2 y)^{\frac{1}{4}}\left(i \pi C_{2}+C_{5}-2 C_{2} \log x+\mathcal{O}_{\log }(x)\right) .
\end{aligned}
$$

To determine the remaining constants we need to compare to the $y \rightarrow 0$ behaviour obtained by the bulk-boundary OPE (2.24),

$$
\begin{aligned}
& \langle\boldsymbol{\mu}(x+i y) \boldsymbol{\omega}(i y)\rangle_{\mathrm{uhp}}^{b} \\
& =(2 y)^{\frac{1}{4}} \cdot \begin{cases}-2 \eta \pi^{\frac{1}{2}} \log (2 y)\langle\mathbf{1}\rangle_{\mathrm{uhp}}^{\mathrm{D}, \eta}+\mathcal{O}_{\log }(y) & ; b=\mathrm{D}, \eta \\
2 \eta \pi^{-\frac{1}{2}}(8 \log x-6 \log y+2 \log 2)\langle\omega(0)\rangle_{\mathrm{uhp}}^{\mathrm{N}, \eta}+\mathcal{O}_{\log }(y) & ; b=\mathrm{N}, \eta .\end{cases}
\end{aligned}
$$

This fixes $C_{2}, C_{4}$ and $C_{5}$ to be

$$
\begin{array}{lll}
b=\mathrm{D}, \eta & : \quad C_{2}=\eta \sqrt{\pi}\langle\mathbf{1}\rangle_{\mathrm{uhp}}^{\mathrm{D}, \eta}, \quad C_{4}=-2 C_{2}, \quad C_{5}=-(i \pi+4 \log 2) C_{2}, \\
b=\mathrm{N}, \eta \quad: & C_{2}=-\frac{2 \eta}{\sqrt{\pi}}\langle\omega(0)\rangle_{\mathrm{uhp}}^{\mathrm{N}, \eta}, & C_{4}=2 C_{2}, \quad C_{5}=-(i \pi+4 \log 2) C_{2} .
\end{array}
$$

Note that substituting the expression for $C_{5}$ simplifies the $x \rightarrow 0$ asymptotics in (5.36) to $-2(2 \log 2+\log x)(2 y)^{\frac{1}{4}} C_{2}+\mathcal{O}_{\log }(x)$. Next we compute the $x \rightarrow 0$ behaviour from the bulk OPE (2.16). One obtains

$$
\begin{aligned}
& \langle\boldsymbol{\mu}(x+i y) \boldsymbol{\omega}(i y)\rangle_{\text {uhp }}^{b} \\
& =-2(2 \log 2+\log x) \cdot(2 y)^{\frac{1}{4}} \cdot \begin{cases}\eta \pi^{\frac{1}{2}}\langle\mathbf{1}\rangle_{\text {uhp }}^{\mathrm{D}, \eta}+\mathcal{O}_{\log }(x) & ; b=\mathrm{D}, \eta \\
-2 \eta \pi^{-\frac{1}{2}}\langle\omega(0)\rangle_{\mathrm{uhp}}^{\mathrm{N}, \eta}+\mathcal{O}_{\log }(x) & ; b=\mathrm{N}, \eta .\end{cases}
\end{aligned}
$$

Again, we find perfect agreement with (15.36) and (5.38). 


\subsubsection{Two $\omega$ fields on the upper half plane}

The calculation for two $\boldsymbol{\omega}$ fields on the upper half plane is more involved because of the large number of free parameters in a four point block with all insertions from $\mathcal{R}_{0}$. The relevant blocks are obtained by applying the projection in appendix E.4 to the fermionic blocks found in appendix E.1. The free parameters can be fixed by requiring first of all that the conformal block gives zero if in (5.26) one chooses one of $\phi$ or $\psi$ to be $\Omega \otimes \Omega+\mathcal{N}$ or $\omega \otimes \Omega-\Omega \otimes \omega+\mathcal{N}$. Second, one has to match the functional form dictated by the conformal block against the asymptotic behaviour determined by the bulk-boundary OPE,

$$
\langle\boldsymbol{\omega}(x+i y) \boldsymbol{\omega}(i y)\rangle_{\mathrm{uhp}}^{b}= \begin{cases}(2 \log (2 y))^{2}\langle\mathbf{1}\rangle_{\mathrm{uhp}}^{\mathrm{D}, \eta}+\mathcal{O}_{\log }\left(y^{2}\right) & ; b=\mathrm{D}, \eta \\ 16(\log (2 y)-2 \log x)\langle\omega(0)\rangle_{\mathrm{uhp}}^{\mathrm{N}, \eta}+\mathcal{O}_{\log }(y) & ; b=\mathrm{N}, \eta .\end{cases}
$$

It turns out that the leading order of the $y \rightarrow 0$ asymptotics as given above is not enough to determine all free parameters of the relevant conformal block. The remaining constants can in principle be fixed by calculating subleading orders of the OPE. However, we will not do this and instead use the $x \rightarrow 0$ behaviour, i.e. the bulk OPE to fix the remaining constants

$$
\langle\boldsymbol{\omega}(x+i y) \boldsymbol{\omega}(i y)\rangle_{\mathrm{uhp}}^{b}= \begin{cases}4 \log x \cdot(2 \log (2 y)-\log x)\langle\mathbf{1}\rangle_{\mathrm{uhp}}^{\mathrm{D}, \eta}+\mathcal{O}_{\log }(x), & ; b=\mathrm{D}, \eta \\ -16 \log x \cdot\langle\omega(0)\rangle_{\mathrm{uhp}}^{\mathrm{N}, \eta}+\mathcal{O}_{\log }(x) & ; b=\mathrm{N}, \eta\end{cases}
$$

Of course, explicitly using the bulk OPE to fix the parameters in the conformal blocks provides a less strong consistency check than the calculation in sections [5.2.1] and [5.2.2 Nonetheless, it is nontrivial that a solution with the correct limits exists.

We carried out the computations outlined above with the help of computer algebra and merely state the result one finds for the correlator,

$$
\langle\boldsymbol{\omega}(x+i y) \boldsymbol{\omega}(i y)\rangle_{\mathrm{uhp}}^{b}= \begin{cases}\left((2 \log (2 y))^{2}-\left(\log \left(1+(2 y / x)^{2}\right)\right)^{2}\right)\langle\mathbf{1}\rangle_{\mathrm{uhp}}^{\mathrm{D}, \eta} & ; b=\mathrm{D}, \eta \\ 8 \log \left(\frac{(2 y / x)^{2}}{x^{2}+(2 y)^{2}}\right)\langle\omega(0)\rangle_{\text {uhp }}^{\mathrm{N}, \eta} & ; b=\mathrm{N}, \eta .\end{cases}
$$

It is easy to see that this reproduces (5.40) and (5.41), while it requires a bit of work to verify that it is in the correct space of conformal blocks, and that it is indeed uniquely fixed by imposing the asymptotics (5.401) and (5.41).

\section{Conclusions}

In this paper we have constructed the boundary theory for the logarithmic triplet theory at $c=-2$ in detail. We started from the four boundary conditions compatible with the free fermion symmetry, and proved that no additional boundary conditions arise when only the triplet algebra is preserved. These four boundary conditions can be labelled by the four irreducible highest weight representations of the triplet algebra, and the corresponding open 
string multiplicities are precisely described by the fusion rules. The open string representations therefore include also indecomposable (but reducible) representations of the triplet algebra.

We have calculated the OPE coefficients of the most interesting boundary operators, namely those which are highest weight with respect to the fermion modes; we have also determined the leading bulk-boundary OPE coefficients. We have found consistent solutions to all factorisation constraints that we have checked. We regard this as very convincing evidence that we have found a consistent boundary theory for the triplet model.

One may expect that a similar approach should also be possible for other 'rational' logarithmic theories, for example for the whole family of $(1, q)$ triplet theories. It would be interesting to understand which features of our analysis generalise directly to these other cases.

One of the motivations of this work was to analyse to which extent logarithmic theories fit into the framework that was developed in 57, 58, 59] for (non-logarithmic) rational conformal field theories. There it is shown that a consistent local conformal field theory can be constructed starting from the algebra of boundary fields for a single boundary condition. Knowledge of the bulk theory or of the other boundary conditions is not required in this construction. Here we have used a 'hybrid' approach: the bulk-boundary OPE coefficients were obtained from the algebra of boundary fields, but the derivation made use of the bulk and boundary spectra since we used the boundary states. It would obviously be interesting to understand whether the above programme can also be applied to this theory. In particular, is it possible to obtain the entire triplet theory starting with the (trivial) algebra structure of the $(\mathrm{D}, \pm)$ boundary that only has the vacuum representation $\mathcal{V}_{0}$ in its spectrum? (In the rational case, the vacuum representation gives rise to the 'Cardy case', i.e. the conformal field theory with charge conjugation modular invariant [57.)

While the algebra on the $(\mathrm{D}, \pm)$ boundary is unique, we found in appendix F.1 a family of non-isomorphic algebras on the representation $\mathcal{R}_{0}$ that makes up the spectrum of the $(\mathrm{N}, \pm)$ boundary. This is something which cannot happen in the rational case. Only one of these algebras was relevant for the triplet theory; it would be interesting to understand the significance of these other algebras. We hope to return to these points in the future.

\section{Acknowledgements}

We thank Jürgen Fuchs, Thomas Quella, Philippe Ruelle and Gérard Watts for conversations and encouragement. The research of MRG was partially supported by the Swiss National Science Foundation and the Marie Curie network 'Constituents, Fundamental Forces and Symmetries of the Universe' (MRTN-CT-2004-005104). The research of IR was partially supported by the EU Research Training Network grants 'Euclid', contract number HPRNCT-2002-00325, and 'Superstring Theory', contract number MRTN-CT-2004-512194. 


\section{A The triplet algebra and its structure constants}

The commutation relations of the triplet algebra are

$$
\begin{aligned}
{\left[L_{m}, L_{n}\right]=} & (m-n) L_{m+n}-\frac{1}{6} m\left(m^{2}-1\right) \delta_{m+n} \\
{\left[L_{m}, W_{n}^{a}\right]=} & (2 m-n) W_{m+n}^{a} \\
{\left[W_{m}^{a}, W_{n}^{b}\right]=} & g^{a b}\left(2(m-n) \Lambda_{m+n}+\frac{1}{20}(m-n)\left(2 m^{2}+2 n^{2}-m n-8\right) L_{m+n}\right. \\
& \left.\quad-\frac{1}{120} m\left(m^{2}-1\right)\left(m^{2}-4\right) \delta_{m+n}\right) \\
& +f_{c}^{a b}\left(\frac{5}{14}\left(2 m^{2}+2 n^{2}-3 m n-4\right) W_{m+n}^{c}+\frac{12}{5} V_{m+n}^{c}\right) .
\end{aligned}
$$

Here $\Lambda=: L^{2}:-3 / 10 \partial^{2} L$ and $V^{a}=: L W^{a}:-3 / 14 \partial^{2} W^{a}$ are quasiprimary normal ordered fields. $g^{a b}$ and $f_{c}^{a b}$ are the metric and structure constants of $s u(2)$. In an orthonormal basis we have $g^{a b}=\delta^{a b}, f_{c}^{a b}=i \epsilon^{a b c}$; for the usual Cartan-Weyl basis that we shall mainly use in this paper, the conventions are

$$
f_{ \pm}^{0 \pm}= \pm 1, \quad f_{0}^{ \pm \mp}= \pm 2, \quad \text { and } \quad g^{00}=1, \quad g^{ \pm \mp}=2 .
$$

The other tensor that is of relevance is $t_{\alpha \beta}^{a}$, whose only non-vanishing components are

$$
t_{ \pm \mp}^{0}=-\frac{1}{2}, \quad t_{ \pm \pm}^{ \pm}= \pm 1
$$

The triplet algebra is only associative (i.e. satisfies the Jacobi identity) if certain null states are divided out [44, 3]. As a consequence, the algebra only exists at $c=-2$.

\section{A.1 The representation $\mathcal{R}_{1}$}

The cyclic states of the indecomposable representation $\mathcal{R}_{1}$ are the doublet of states at $h=1$

$$
\phi^{ \pm}=\chi_{-1}^{ \pm} \omega
$$

These states are not highest weight; instead one has

$$
\begin{aligned}
L_{1} \phi^{ \pm} & =\chi_{0}^{ \pm} \omega=\xi^{ \pm} \\
L_{-1} \xi^{ \pm} & =-\chi_{-1}^{ \pm} \Omega=-\psi^{ \pm} \\
L_{0} \phi^{ \pm} & =\phi^{ \pm}+\psi^{ \pm} .
\end{aligned}
$$

To verify this note that the Virasoro modes are expressed in terms of the fermionic modes as

$$
L_{m}=\sum_{k=-\infty}^{-1} \frac{1}{2} d_{\alpha \beta} \chi_{k}^{\alpha} \chi_{m-k}^{\beta}+\sum_{k=0}^{\infty} \frac{1}{2} d_{\alpha \beta} \chi_{m-k}^{\alpha} \chi_{k}^{\beta} .
$$

The action of the modes $W_{n}^{a}$ can also be easily determined [3]. The representation $\mathcal{R}_{1}$ is again indecomposable but reducible; its cyclic vector $\phi^{ \pm}$is not highest weight, but the spectrum of $L_{0}$ is bounded from below [46, 3], and thus $\mathcal{R}_{1}$ is a highest weight representation. 


\section{B Theta functions}

For the description of the free fermionic theory it is convenient to introduce the functions $f_{i}$ that are defined as follows $\left(q=e^{2 \pi i \tau}\right)$

$$
\begin{aligned}
& f_{1}(q)=q^{\frac{1}{24}} \prod_{n=1}^{\infty}\left(1-q^{n}\right), \\
& f_{2}(q)=\sqrt{2} q^{\frac{1}{24}} \prod_{n=1}^{\infty}\left(1+q^{n}\right), \\
& f_{3}(q)=q^{-\frac{1}{48}} \prod_{n=1}^{\infty}\left(1+q^{(n-1 / 2)}\right), \\
& f_{4}(q)=q^{-\frac{1}{48}} \prod_{n=1}^{\infty}\left(1-q^{(n-1 / 2)}\right) .
\end{aligned}
$$

Under the modular S-transformation, $\tau \mapsto-1 / \tau, f_{2}$ and $f_{4}$ get interchanged, while $f_{3}$ is invariant. The function $f_{1}(q)$ agrees with the Dedekind eta-function, and transforms as

$$
f_{1}(-1 / \tau)=\sqrt{-i \tau} f_{1}(\tau)
$$

\section{Field insertions and local coordinates}

To define correlation functions on Riemann surfaces without a preferred global coordinate one needs a formulation of field insertions which includes a local coordinate system around the insertion point. Below we first introduce the relevant notation for bulk and boundary fields, and then use this to compute two-point functions on the Riemann sphere and the upper half plane.

\section{C.1 Bulk fields and local coordinates}

To define correlation functions on Riemann surfaces other than the complex plane, one needs a formulation of field insertions that does not rely on the existence of a global coordinate. Let $\mathcal{H}^{\text {bulk }}$ be the space of bulk states of a conformal field theory, and denote by $D_{\varepsilon}$ the disc $\{z \in \mathbb{C}|| z \mid<\varepsilon\}$. A bulk field on a Riemann surface $\Sigma$ is a pair

$$
[\varphi, v] ; \quad v \in \mathcal{H}^{\text {bulk }}, \quad \varphi: D_{\varepsilon} \rightarrow \Sigma \text { injective and holomorphic } .
$$

In words, $[\varphi, v]$ is a field labelled by $v$ inserted at the point $\varphi(0)$ of $\Sigma$ with a choice of local coordinates $\varphi$ around the insertion point. A correlator of several bulk fields on a surface $\Sigma$ is then written as

$$
\left\langle\left[\varphi_{1}, v_{1}\right]\left[\varphi_{2}, v_{2}\right] \cdots\left[\varphi_{n}, v_{n}\right]\right\rangle_{\Sigma} .
$$

The ordering of the bulk fields can be chosen at will and has no influence on the correlator. Isomorphic Riemann surfaces with bulk fields are required to result in equal correlators. 
That is, given an isomorphism $f: \Sigma \rightarrow \Sigma^{\prime}$,

$$
\left\langle\left[\varphi_{1}, v_{1}\right] \cdots\left[\varphi_{n}, v_{n}\right]\right\rangle_{\Sigma}=\left\langle\left[f \circ \varphi_{1}, v_{1}\right] \cdots\left[f \circ \varphi_{n}, v_{n}\right]\right\rangle_{\Sigma^{\prime}} .
$$

This holds in particular if $\Sigma=\Sigma^{\prime}$.

The description (C.1) of bulk fields is in fact quite redundant because a change in the local coordinates can be traded for a change of the vector $v$ without affecting the value of a correlator. This can be captured by introducing an equivalence relation on bulk fields, namely we set, for any injective holomorphic function $f: D_{\varepsilon} \rightarrow D_{\varepsilon}$ with $f(0)=0$,

$$
[\varphi \circ f, v] \sim[\varphi, \rho(f) v], \quad \text { where } \rho(f)=\left(a_{0}\right)^{L_{0}}\left(a_{0}^{*}\right)^{\bar{L}_{0}} e^{\sum_{m=1}^{\infty}\left(a_{m} L_{m}+a_{m}^{*} \bar{L}_{m}\right)} .
$$

The $f$-dependent constants $a_{m}$ are determined by matching coefficients of

$$
a_{0} \exp \left(\sum_{m=1}^{\infty} a_{m} t^{m+1} \frac{\partial}{\partial t}\right) t=\sum_{k=0}^{\infty} \frac{1}{k !} f^{(k)}(0) t^{k}, \quad f^{(k)}(t)=\frac{\partial^{k}}{\partial t^{k}} f(t)
$$

order by order in $t$. The first three coefficients are

$$
a_{0}=f^{\prime}, \quad a_{1}=\frac{1}{2} \frac{f^{\prime \prime}}{f^{\prime}}, \quad a_{2}=\frac{1}{6} \frac{f^{\prime \prime \prime}}{f^{\prime}}-\frac{1}{4}\left(\frac{f^{\prime \prime}}{f^{\prime}}\right)^{2},
$$

with all derivatives evaluated at zero. Replacing a field $[\varphi, v]$ by an equivalent field $[\psi, w] \sim$ $[\varphi, v]$ does not affect the value of a correlator.

There is no closed expression for the coefficients $a_{m}$ in (C.5), but they can be computed by a recursive formula [60]. For more details on the action of the group of local coordinate changes and the relation to the definition of conformal blocks, see section 6.3.1 of [61.

For (C.4) to be well-defined, the conformal field theory has to fulfil two further requirements. First, for every element $v \in \mathcal{H}^{\text {bulk }}$ there has to exists an $M$ such that $v$ is annihilated by all $L_{m}, \bar{L}_{m}$ with $m>M$. Otherwise $\rho(f) u$ can result in an infinite sum and is no longer an element of $\mathcal{H}^{\text {bulk }}$. Second, consider the family of local coordinates $f_{t}(\zeta)=e^{i t} \zeta$. One has $\rho\left(f_{t}\right)=e^{i t\left(L_{0}-\bar{L}_{0}\right)}$ and continuously varying $t$ from 0 to $2 \pi$ in $\left[\varphi \circ f_{t}, u\right] \sim\left[\varphi, \rho\left(f_{t}\right) u\right]$ results in $[\varphi, u] \sim\left[\varphi, e^{2 \pi i\left(L_{0}-\bar{L}_{0}\right)} u\right]$, which can only be true inside every correlator if $e^{2 \pi i\left(L_{0}-\bar{L}_{0}\right)} u=u$. This has to hold for all $u \in \mathcal{H}^{\text {bulk }}$.

We can define mode integrals of a holomorphic field $K \in \mathcal{H}^{\text {bulk }}$ of weight $h$ as

$$
\left\langle\cdots\left[\varphi, K_{m} v\right] \cdots\right\rangle=\oint w^{m+h-1}\langle\cdots[\varphi \circ(\zeta \mapsto \zeta+w), K][\varphi, v] \cdots\rangle \frac{d w}{2 \pi i},
$$

where the contour integral is along a small circle around zero that lies entirely in $D_{\varepsilon}$. By analytic continuation, the contour integral can then be deformed on the entire Riemann surface $\Sigma$. Contour integrals of anti-holomorphic fields are defined similarly.

\section{C.2 Boundary fields and local coordinates}

For boundary fields the discussion is analogous to the one for bulk fields. Let $\mathcal{H}^{\text {bnd }}$ be the space of states of a boundary conformal field theory, and denote by $H_{\varepsilon}$ the half-disc 
$\left\{z \in D_{\varepsilon} \mid \operatorname{Im} z \geq 0\right\}$. A boundary field on a Riemann surface $\Sigma$ is a pair $[\varphi, v]$ with $v \in \mathcal{H}^{\text {bnd }}$ and $\varphi: H_{\varepsilon} \rightarrow \Sigma$ an injective, holomorphic and boundary preserving map. By boundary preserving we mean that $\varphi$ maps the points $H_{\varepsilon} \cap \mathbb{R}$ to $\partial \Sigma$.

For correlators the notation (C.2) is used, and the identity (C.3) applies also in the presence of boundary fields. As for bulk fields, the description of boundary fields is redundant, and a change in the local coordinates can be traded for a change of the vector $v$ without affecting the value of a correlator. The corresponding equivalence relation is, for any injective holomorphic function $f: H_{\varepsilon} \rightarrow H_{\varepsilon}$ with $f(0)=0$,

$$
[\varphi \circ f, v] \sim[\varphi, \rho(f) v], \quad \text { where } \quad \rho(f)=\left(a_{0}\right)^{L_{0}} e^{\sum_{m=1}^{\infty} a_{m} L_{m}} .
$$

The coefficients $a_{m}$ are the same as in (C.5).

\section{C.3 Two-point function on the Riemann sphere}

Consider the Riemann sphere $\mathbb{P}^{1}$ and let $[z: w]$ be the homogeneous coordinates. Define the local coordinates $\varphi_{a}(\zeta)=[\zeta+a: 1]$ for $a \in \mathbb{C}$, and $\varphi^{\infty}(\zeta)=[1: \zeta]$. Using the correlator of two bulk fields on the sphere, we define a bilinear form $B: \mathcal{H}^{\text {bulk }} \times \mathcal{H}^{\text {bulk }} \rightarrow \mathbb{C}$ as

$$
B(u, v)=\left\langle\left[\varphi^{\infty}, u\right]\left[\varphi_{0}, v\right]\right\rangle_{\mathbb{P}^{1}} .
$$

Composing with the isomorphism $f([z: w])=[w: z]$ from $\mathbb{P}^{1}$ to itself and making use of the property (C.3) we find

$$
B(u, v)=\left\langle\left[f \circ \varphi^{\infty}, u\right]\left[f \circ \varphi_{0}, v\right]\right\rangle_{\mathbb{P}^{1}}=\left\langle\left[\varphi_{0}, u\right]\left[\varphi^{\infty}, v\right]\right\rangle_{\mathbb{P}^{1}}=B(v, u),
$$

i.e. $B$ is symmetric. Furthermore, one requires $B$ to be non-degenerate, as fields $u$ for which $B(u, \cdot)$ is identically zero would vanish inside all correlators and one could pass to a quotient of $\mathcal{H}^{\text {bulk }}$.

In terms of the contour integration mentioned in the previous section, it is not difficult to verify that for Virasoro primary holomorphic and anti-holomorphic fields $K$ and $\bar{K}$ of weights $h$ and $\bar{h}$ we have

$$
B\left(K_{m} u, v\right)=(-1)^{h} B\left(u, K_{-m} v\right) \quad \text { and } \quad B\left(\bar{K}_{m} u, v\right)=(-1)^{\bar{h}} B\left(u, \bar{K}_{-m} v\right) .
$$

For the symplectic fermion theory, this identity shows that we can shift pairs of fermion modes from one argument to the other,

$$
B\left(\chi_{m}^{\alpha} \chi_{n}^{\beta} u, v\right)=B\left(u, \chi_{-n}^{-\beta} \chi_{-m}^{-\alpha} v\right), \quad B\left(\bar{\chi}_{m}^{\alpha} \bar{\chi}_{n}^{\beta} u, v\right)=B\left(u, \bar{\chi}_{-n}^{-\beta} \bar{\chi}_{-m}^{-\alpha} v\right) .
$$

For a correlator on $\mathbb{P}^{1}$ where all fields are of the form $\left[\varphi_{z}, \phi\right]$, we will also use the more conventional notation $\phi(z)$ to denote the fields,

$$
\left\langle\left[\varphi_{z_{1}}, \phi_{1}\right] \cdots\left[\varphi_{z_{n}}, \phi_{n}\right]\right\rangle_{\mathbb{P}^{1}}=\left\langle\phi_{1}\left(z_{1}\right) \cdots \phi_{n}\left(z_{n}\right)\right\rangle_{\mathbb{P}^{1}} .
$$

In this notation, the bilinear form $B(\cdot, \cdot)$ determines the two-point function $\left\langle\phi(a) \phi^{\prime}(b)\right\rangle_{\mathbb{P} 1}$ as follows. The Möbius transformation $M([z: w])=[z-b w: z-a w]$ takes $[a: 1]$ to $[1: 0]$ and $[b: 1]$ to $[0: 1]$. Note that

$$
M \circ \varphi_{a}(\zeta)=[\zeta+a-b: \zeta]=\varphi^{\infty} \circ f(\zeta), \quad \text { where } \quad f(\zeta)=\frac{\zeta}{\zeta+a-b}
$$


and similarly $M \circ \varphi_{b}=\varphi_{0} \circ g$ with $g(\zeta)=\frac{\zeta}{\zeta+b-a}$. Using relation (C.3) one computes

$$
\begin{aligned}
& \left\langle\phi(a) \phi^{\prime}(b)\right\rangle_{\mathbb{P}^{1}}=\left\langle\left[M \circ \varphi_{a}, \phi\right]\left[M \circ \varphi_{b}, \phi^{\prime}\right]\right\rangle_{\mathbb{P}^{1}} \\
& =\left\langle\left[\varphi^{\infty} \circ f, \phi\right]\left[\varphi_{0} \circ g, \phi^{\prime}\right]\right\rangle_{\mathbb{P}^{1}}=B\left(\rho(f) \phi, \rho(g) \phi^{\prime}\right) .
\end{aligned}
$$

Let us evaluate this expression for states $\boldsymbol{\omega}$ and $\Omega$ of the space of bulk states $\mathcal{H}^{\text {bulk }}$ of the triplet theory. Since $L_{0} \boldsymbol{\omega}=\boldsymbol{\Omega}=\bar{L}_{0} \boldsymbol{\omega}$ and $L_{0} \boldsymbol{\Omega}=0=\bar{L}_{0} \boldsymbol{\Omega}$ we have

$$
B(\boldsymbol{\Omega}, \boldsymbol{\Omega})=B\left(L_{0} \boldsymbol{\omega}, \boldsymbol{\Omega}\right)=B\left(\boldsymbol{\omega}, L_{0} \boldsymbol{\Omega}\right)=0
$$

By similar reasoning one sees that $B(\boldsymbol{\Omega}, \cdot)$ can only be non-vanishing on the subspace $\mathbb{C} \boldsymbol{\Omega} \oplus$ $\mathbb{C} \boldsymbol{\omega}$ of $\mathcal{H}^{\text {bulk }}$. Non-degeneracy of $B$ then requires $B(\boldsymbol{\Omega}, \boldsymbol{\omega}) \neq 0$. If also $B(\boldsymbol{\omega}, \boldsymbol{\omega}) \neq 0$ we redefine $\boldsymbol{\omega} \rightarrow \boldsymbol{\omega}-\frac{B(\omega, \omega)}{2 B(\Omega, \omega)} \boldsymbol{\Omega}$ so that $B(\boldsymbol{\omega}, \boldsymbol{\omega})=0$. We take the correlators on $\mathbb{P}^{1}$ to be normalised such that $B(\boldsymbol{\omega}, \boldsymbol{\Omega})=-1$, so that altogether we get

$$
B(\boldsymbol{\Omega}, \boldsymbol{\Omega})=B(\boldsymbol{\omega}, \boldsymbol{\omega})=0, \quad B(\boldsymbol{\omega}, \boldsymbol{\Omega})=-1 .
$$

The next ingredient we need in (C.15) is $\rho(f) u$. Combining (C.4) and (C.14) with $L_{0} \boldsymbol{\omega}=\bar{L}_{0} \boldsymbol{\omega}$ one finds

$$
\rho(f) \boldsymbol{\Omega}=\boldsymbol{\Omega}, \quad \rho(f) \boldsymbol{\omega}=e^{\log \left(\left|f^{\prime}\right|^{2}\right) L_{0}} \boldsymbol{\omega}=\boldsymbol{\omega}+\log \left(|a-b|^{-2}\right) \boldsymbol{\Omega} .
$$

For $\rho(g)$ one finds the same result, and the two-point functions on $\mathbb{P}^{1}$ are thus equal to $\langle\boldsymbol{\Omega}(a) \boldsymbol{\Omega}(b)\rangle_{\mathbb{P}^{1}}=\langle\mathbf{1}\rangle_{\mathbb{P}^{1}}=0$ and

$$
\langle\boldsymbol{\omega}(a) \boldsymbol{\omega}(b)\rangle_{\mathbb{P}^{1}}=4 \log |a-b|, \quad\langle\boldsymbol{\omega}(a) \boldsymbol{\Omega}(b)\rangle_{\mathbb{P}^{1}}=\langle\boldsymbol{\omega}(a)\rangle_{\mathbb{P}^{1}}=-1,
$$

where we used that $\boldsymbol{\Omega}$ is the identity field, and $\langle\mathbf{1}\rangle$ denotes a correlator with no field insertions. We are still free to choose the normalisation of the bulk field $\boldsymbol{\mu}$. We demand that $B(\boldsymbol{\mu}, \boldsymbol{\mu})=1$ which results in the two-point function

$$
\langle\boldsymbol{\mu}(a) \boldsymbol{\mu}(b)\rangle_{\mathbb{P}^{1}}=|a-b|^{\frac{1}{2}} .
$$

If we combine this with the one-point function of $\boldsymbol{\omega}$ in (C.19), we see that equivalently we can demand the OPE of $\boldsymbol{\mu}$ with itself to be of the form $\boldsymbol{\mu}(z) \boldsymbol{\mu}(0)=|z|^{\frac{1}{2}}(-\boldsymbol{\omega}(0)+($ other fields $))$, which is indeed the convention used in (2.16).

\section{C.4 Two-point function on the upper half plane}

Let $\mathbb{U}$ be the upper half plane together with the point at infinity. We will represent $\mathbb{U}$ as the quotient of $\mathbb{P}^{1}$ by the anti-holomorphic involution $\iota:[z: w] \mapsto\left[z^{*}: w^{*}\right]$. For points of $\mathbb{U}$ we also use the notation $[z: w]$. The fixed points of $\iota$, which form the boundary of $\mathbb{U}$, are $[r: 1]$ for $r \in \mathbb{R}$ together with [1:0]. We fix an orientation on $\mathbb{U}$ by demanding the map $z \mapsto[z: 1]$ from the upper half plane to $\mathbb{U}$ to be orientation preserving.

Let $\tilde{\varphi}_{a}: H_{\varepsilon} \rightarrow \mathbb{U}, a \in \mathbb{R}$, and $\tilde{\varphi}^{\infty}: H_{\varepsilon} \rightarrow \mathbb{U}$ be the local coordinates $\tilde{\varphi}_{a}(\zeta)=[\zeta+a: 1]$ and $\tilde{\varphi}^{\infty}(\zeta)=[1:-\zeta]$. One checks that $\tilde{\varphi}_{a}$ and $\tilde{\varphi}^{\infty}$ are orientation and boundary preserving (while $\zeta \mapsto[1: \zeta]$ would not be orientation preserving as a map from $H_{\varepsilon}$ to $\mathbb{U}$ ). 
Similar as for bulk fields, the two-point function on $\mathbb{U}$ gives rise to a bilinear pairing $b$ on the space of boundary fields $\mathcal{H}^{\text {bnd }}$ for a given boundary condition,

$$
b(u, v)=\left\langle\left[\tilde{\varphi}^{\infty}, u\right]\left[\tilde{\varphi}_{0}, v\right]\right\rangle_{\mathbb{U}} .
$$

Applying the orientation preserving isomorphism $[z: w] \mapsto[w:-z]$ of $\mathbb{U}$ shows that $b$ is symmetric, $b(u, v)=b(v, u)$.

For the triplet theory, consider a boundary condition such that $\mathcal{H}^{\text {bnd }}=\mathcal{R}_{0}$. We then always define the boundary field $\omega$ such that $L_{0} \omega=\Omega$ and such that $b(\omega, \omega)=0$. That $b(\Omega, \Omega)=0$ follows in the same way as it did for the corresponding bulk fields. Using appropriate Möbius transformations and (C.8), the relation between the two-point function of $\omega$ and the constant $\langle\omega(0)\rangle_{\mathbb{U}}=b(\Omega, \omega)$ one finds is, for $x, y \in \mathbb{R}, x>y$,

$$
\langle\omega(x) \omega(y)\rangle_{\mathbb{U}} \equiv\left\langle\left[\tilde{\varphi}_{x}, \omega\right]\left[\tilde{\varphi}_{y}, \omega\right]\right\rangle_{\mathbb{U}}=-2 \log (x-y)\langle\omega(0)\rangle_{\mathbb{U}}
$$

\section{Intertwiners for fermion representations}

For the purposes of this paper we will need two of the indecomposable representations of the symplectic fermion mode algebra, namely $\mathcal{H}_{\omega}$ and $\mathcal{H}_{\mu}$. Recall from section 2 that $\mathcal{H}_{\omega}$ is an untwisted representation generated by a vector $\omega$ and characterised by the properties that $\chi_{m}^{\alpha} \omega=0$ for $m \in \mathbb{Z}_{>0}$ and that the four vectors $\omega, \chi_{0}^{+} \omega, \chi_{0}^{-} \omega, \chi_{0}^{+} \chi_{0}^{-} \omega$ are linearly independent. The representation $\mathcal{H}_{\mu}$ is twisted and generated by a vector $\mu$ which obeys $\chi_{m}^{\alpha} \mu=0$ for $m \in \frac{1}{2}+\mathbb{Z}_{\geq 0}$.

Denote by $\mathcal{H}_{\omega}^{0}$ the subspace of $\mathcal{H}_{\omega}$ spanned by $\left\{\omega, \chi_{0}^{+} \omega, \chi_{0}^{-} \omega, \chi_{0}^{+} \chi_{0}^{-} \omega\right\}$, i.e. the space of lowest generalised $L_{0}$-weight. Let further $Q: \mathcal{H}_{\omega} \rightarrow \mathcal{H}_{\omega}^{0}$ be the projector onto $\mathcal{H}_{\omega}^{0}$. The parity operator on $\mathcal{H}_{\omega}$ and $\mathcal{H}_{\mu}$ is denoted by $(-1)^{F}$. It is defined by $(-1)^{F} \chi_{m}^{\alpha}=-\chi_{m}^{\alpha}(-1)^{F}$ and $(-1)^{F} \omega=\omega,(-1)^{F} \mu=\mu$. The representations $\mathcal{H}_{\omega}$ and $\mathcal{H}_{\mu}$ are graded by generalised $L_{0}$ weight, and we will write $\mathcal{H}_{\omega}^{\vee}$ and $\mathcal{H}_{\mu}^{\vee}$ for their graded duals.

In the following we shall consider three types of intertwiners between these representations.

\section{D.1 Intertwiners of type $\mathcal{H}_{\omega} \times \mathcal{H}_{\omega} \rightarrow \mathcal{H}_{\omega}$}

The (super) vertex algebra built from the symplectic fermions (as well as its bosonic subalgebra, the triplet vertex operator algebra) are described in [47. Since the action of $L_{0}$ on $\mathcal{H}_{\omega}$ is not diagonalisable, an intertwiner of type $\mathcal{H}_{\omega} \times \mathcal{H}_{\omega} \rightarrow \mathcal{H}_{\omega}$ can be logarithmic. Logarithmic intertwiners of representations of vertex algebras have been treated in [27, 29]. An intertwiner $V$ of type $\mathcal{H}_{\omega} \times \mathcal{H}_{\omega} \rightarrow \mathcal{H}_{\omega}$ is a linear map

$$
V(\cdot, z): \mathcal{H}_{\omega} \longrightarrow L\left(\mathcal{H}_{\omega}, \mathcal{H}_{\omega}\right) \llbracket z^{ \pm 1}, \log z \rrbracket
$$

which is compatible with the action of the fermion modes, see [27, 29] for details. Here $L(U, V)$ denotes the space of linear maps between two vector spaces $U$ and $V$, and by $W \llbracket x, y, \ldots \rrbracket$ we denote the space of formal power series in $x, y, \ldots$ with coefficients in $W$. 
To be completely precise, we should write $\ell_{z}$ instead of $\log z$ and treat it as an independent formal variable with certain properties, but this distinction will not be important below.

The statement that $V(\cdot, z)$ is an intertwiner is equivalent to the identities, for $u \in \mathcal{H}_{\omega}$, $a \in \mathcal{H}_{\omega}^{0}$ and $m \in \mathbb{Z}$

$$
\begin{aligned}
V\left(\chi_{m}^{\alpha} u, z\right) & =\sum_{k=0}^{\infty}\left(\begin{array}{c}
m \\
k
\end{array}\right)\left((-z)^{k} \chi_{m-k}^{\alpha} V(u, z)-(-z)^{m-k} V\left((-1)^{F} u, z\right) \chi_{k}^{\alpha}\right), \\
\chi_{m}^{\alpha} V(a, z) & =z^{m} V\left(\chi_{0}^{\alpha} a, z\right)+V\left((-1)^{F} a, z\right) \chi_{m}^{\alpha}, \\
{\left[L_{-1}, V(u, z)\right] } & =\frac{\partial}{\partial z} V(u, z) .
\end{aligned}
$$

These commutation relation for the modes can be derived from the usual contour deformation arguments. A useful relation which is a direct consequence of (D.2) is

$$
\left[\chi_{m}^{\alpha}, V(\omega, z)\right]=z^{m}\left[\chi_{0}^{\alpha}, V(\omega, z)\right] .
$$

For $u^{*} \in \mathcal{H}_{\omega}^{\vee}$ and $v \in \mathcal{H}_{\omega}$ denote by $\left\langle u^{*}, v\right\rangle$ the canonical pairing. Using the relations (D.2), the expression $\left\langle u^{*}, V(v, z) w\right\rangle$, for $u^{*} \in \mathcal{H}_{\omega}^{\vee}, v, w \in \mathcal{H}_{\omega}$, can be reduced to a finite sum involving only terms of the form $\left\langle a^{*}, V(\omega, z) b\right\rangle z^{m}(\log z)^{n}$, where $a^{*} \in\left(\mathcal{H}_{\omega}^{0}\right)^{*}, b \in \mathcal{H}_{\omega}^{0}$ and $m, n \in \mathbb{Z}$.

Abbreviate by $V^{0}(u, z)=Q V(u, z) Q$ the restriction of $V(u, z)$ to $\mathcal{H}_{\omega}^{0}$. Then the above reasoning implies that $V(u, z)$ is uniquely determined by $V^{0}(\omega, z)$ and the relations (D.2). By analysing the action of $L_{0}$ or by directly using [29, proposition 3.9] we see,

$$
V^{0}(\omega, z)=A+B \log z+C(\log z)^{2}+D(\log z)^{3},
$$

where $A, B, C, D \in L\left(\mathcal{H}_{\omega}^{0}, \mathcal{H}_{\omega}^{0}\right)$. Inserting the expression A.6 of $L_{-1}$ in terms of fermion modes into the relation $Q\left[L_{-1}, V^{0}(\omega, z)\right] Q=\frac{\partial}{\partial z} V^{0}(\omega, z)$ gives the condition

$$
\begin{aligned}
& z^{-1}\left(\chi_{0}^{-} V^{0}(\omega, z) \chi_{0}^{+}-\chi_{0}^{+} V^{0}(\omega, z) \chi_{0}^{-}+2 V^{0}(\omega, z) \chi_{0}^{+} \chi_{0}^{-}\right) \\
& =z^{-1} B+2 C z^{-1} \log z+3 D z^{-1}(\log z)^{2}
\end{aligned}
$$

This allows to fix $B, C, D$ in terms of $A$. Thus giving an element $A \in L\left(\mathcal{H}_{\omega}^{0}, \mathcal{H}_{\omega}^{0}\right)$ determines uniquely an intertwiner of type $\mathcal{H}_{\omega} \times \mathcal{H}_{\omega} \rightarrow \mathcal{H}_{\omega}$. We will denote this intertwiner by $V_{A}(\cdot, z)$. Its action on $\mathcal{H}_{\omega}^{0}$ is easily found to be (recall that $\Omega=-\chi_{0}^{+} \chi_{0}^{-} \omega$ )

$$
\begin{aligned}
V_{A}^{0}(\omega, z) & =A+\left(\chi_{0}^{-} A \chi_{0}^{+}-\chi_{0}^{+} A \chi_{0}^{-}+2 A \chi_{0}^{+} \chi_{0}^{-}\right) \log z-\chi_{0}^{+} \chi_{0}^{-} A \chi_{0}^{+} \chi_{0}^{-}(\log z)^{2}, \\
V_{A}^{0}\left(\chi_{0}^{\alpha} \omega, z\right) & =\chi_{0}^{\alpha} A-A \chi_{0}^{\alpha}+\left(\chi_{0}^{+} \chi_{0}^{-} A \chi_{0}^{\alpha}+\chi_{0}^{\alpha} A \chi_{0}^{+} \chi_{0}^{-}\right) \log z \\
V_{A}^{0}(\Omega, z) & =-\chi_{0}^{+} \chi_{0}^{-} A+\chi_{0}^{+} A \chi_{0}^{-}-\chi_{0}^{-} A \chi_{0}^{+}-A \chi_{0}^{+} \chi_{0}^{-} .
\end{aligned}
$$

Denote by $\operatorname{Hom}\left(\mathcal{H}_{\omega} \otimes \mathcal{H}_{\omega}, \mathcal{H}_{\omega}\right)$ the space of intertwiners of type $\mathcal{H}_{\omega} \times \mathcal{H}_{\omega} \rightarrow \mathcal{H}_{\omega}$. The above arguments show that this space is at most 16 dimensional (which is the dimension of $L\left(\mathcal{H}_{\omega}^{0}, \mathcal{H}_{\omega}^{0}\right)$ ). Since $\mathcal{H}_{\omega}$ does not contain null-vectors (with respect to the fermion modes), it is plausible that the dimension is exactly 16 , but we have not pursued this further. 


\section{D.2 Intertwiners of type $\mathcal{H}_{\mu} \times \mathcal{H}_{\mu} \rightarrow \mathcal{H}_{\omega}$ and $\mathcal{H}_{\mu} \times \mathcal{H}_{\omega} \rightarrow \mathcal{H}_{\mu}$}

Let $U$ be an intertwiner of type $\mathcal{H}_{\mu} \times \mathcal{H}_{\mu} \rightarrow \mathcal{H}_{\omega}$,

$$
U(\cdot, z): \mathcal{H}_{\mu} \longrightarrow z^{\frac{1}{4}} L\left(\mathcal{H}_{\mu}, \mathcal{H}_{\omega}\right) \llbracket z^{ \pm 1}, \log z \rrbracket .
$$

The condition analogous to (D.2) is more involved for twisted representations, see e.g. 62 63. 64. We will just need the relation

$$
\chi_{m}^{\alpha} U(\mu, z) \mu=-\sum_{k=1}^{\infty}\left(\begin{array}{c}
\frac{1}{2} \\
k
\end{array}\right)(-z)^{k} \chi_{m-k}^{\alpha} U(\mu, z) \mu=\left(\frac{z}{2} \chi_{m-1}^{\alpha}+\frac{z^{2}}{8} \chi_{m-2}^{\alpha}+\cdots\right) U(\mu, z) \mu,
$$

which is valid for $m \geq 1$. It can be derived by expanding

$$
0=\oint_{\mathcal{C}_{\infty}} \frac{d \zeta}{2 \pi i} \sqrt{\zeta(\zeta-z)} \zeta^{m-1} \chi^{\alpha}(\zeta) U(\mu, z) \mu
$$

in powers of $\zeta^{-1}$. The contour $\mathcal{C}_{\infty}$ is a large anti-clockwise oriented circle containing the points 0 and $z$. In any case, the expression $\left\langle u^{*}, U(v, z) w\right\rangle$ for $u^{*} \in \mathcal{H}_{\omega}^{\vee}$ and $v, w \in \mathcal{H}_{\mu}$ is uniquely determined once we know $Q U(\mu, z) \mu$. Compatibility with the action of $L_{0}$ forces

$$
Q U(\mu, z) \mu=a+b \log z
$$

for $a, b \in \mathcal{H}_{\omega}^{0}$. As before, $b$ is fixed in terms of $a$ by exploiting the condition $\left[L_{-1}, U(\mu, z)\right]=$ $\frac{\partial}{\partial z} U(\mu, z)$. This is easiest done in the form $\left[2 z^{-1} L_{0}-z^{-2} L_{1}, U(\mu, z)\right]=\frac{\partial}{\partial z} U(\mu, z)$, together with (D.8). Denoting the intertwiner determined by $a \in \mathcal{H}_{\omega}^{0}$ by $U_{a}(\cdot, z)$ we find

$$
Q U_{a}(\mu, z) \mu=z^{\frac{1}{4}}\left(a-\log z \cdot \chi_{0}^{+} \chi_{0}^{-} a\right) .
$$

It also follows that the dimension of $\operatorname{Hom}\left(\mathcal{H}_{\mu} \otimes \mathcal{H}_{\mu}, \mathcal{H}_{\omega}\right)$ is at most four.

Next, let $\widetilde{U}$ be an intertwiner of type $\mathcal{H}_{\mu} \times \mathcal{H}_{\omega} \rightarrow \mathcal{H}_{\mu}$,

$$
\widetilde{U}(\cdot, z): \mathcal{H}_{\mu} \longrightarrow L\left(\mathcal{H}_{\omega}, \mathcal{H}_{\mu}\right) \llbracket z^{ \pm 1}, \log z \rrbracket .
$$

Let $\mu^{*} \in \mathcal{H}_{\mu}^{\vee}$ be the unique linear form such that $\left\langle\mu^{*}, \mu\right\rangle=1$ and $\left\langle\mu^{*}, v\right\rangle=0$ for any $v \in \mathcal{H}_{\mu}$ with $L_{0}$-weight greater than $-\frac{1}{8}$. The relation corresponding to (D.8) reads

$$
\mu^{*} \circ \widetilde{U}(\mu, z) \chi_{m}^{\alpha}=-\sum_{k=1}^{\infty}\left(\begin{array}{l}
\frac{1}{2} \\
k
\end{array}\right)(-z)^{-k} \mu^{*} \circ \widetilde{U}(\mu, z) \chi_{m+k}^{\alpha}
$$

which is valid for $m \leq-1$. The expression $\left\langle u^{*}, \widetilde{U}(v, z) p\right\rangle$ for $u^{*} \in \mathcal{H}_{\mu}^{\vee}, v \in \mathcal{H}_{\mu}$ and $p \in \mathcal{H}_{\omega}$ is then uniquely determined once we know $\mu^{*} \circ \widetilde{U}(\mu, z) Q$. Compatibility with the action of $L_{0}$ and $L_{-1}$ shows that an intertwiner of type $\mathcal{H}_{\mu} \times \mathcal{H}_{\mu} \rightarrow \mathcal{H}_{\omega}$ is determined by an element $\varphi \in\left(\mathcal{H}_{\omega}^{0}\right)^{*}$. The corresponding intertwiner is denoted by $\widetilde{U}_{\varphi}(\cdot, z)$ and obeys

$$
\mu^{*} \circ \widetilde{U}_{\varphi}(\mu, z) Q=\varphi+\log z \cdot \varphi \chi_{0}^{+} \chi_{0}^{-} .
$$

The dimension of $\operatorname{Hom}\left(\mathcal{H}_{\mu} \otimes \mathcal{H}_{\omega}, \mathcal{H}_{\mu}\right)$ is therefore also at most four. 


\section{E Fermionic four-point blocks}

For the computations in the main text we need various bosonic four-point blocks, which all derive from three basic fermionic four-point blocks, namely the blocks involving four times $\mathcal{H}_{\omega}$, two times $\mathcal{H}_{\omega}$ and two times $\mathcal{H}_{\mu}$, and four times $\mathcal{H}_{\mu}$.

\section{E.1 The $\omega \omega \omega \omega$-block}

We need to compute

$$
Q V_{A}(a, z)(-1)^{\varepsilon F} V_{B}(b, w) Q \in L\left(\mathcal{H}_{\omega}^{0}, \mathcal{H}_{\omega}^{0}\right) \llbracket z^{ \pm 1}, w^{ \pm 1}, \log z, \log w \rrbracket,
$$

where $A, B \in L\left(\mathcal{H}_{\omega}^{0}, \mathcal{H}_{\omega}^{0}\right), a, b \in \mathcal{H}_{\omega}^{0}$ and $\varepsilon \in\{0,1\}$. We will do this by explicitly summing over all intermediate states. This is possible because

$$
\chi_{k}^{\alpha} \chi_{l}^{\beta} \chi_{m}^{\gamma} V_{B}(b, w) Q=0
$$

for all $m, n, k \geq 1$, as follows by applying (D.2) three times and noting that a product of three fermion zero modes is always zero.

One can verify that the identity map on $\mathcal{H}_{\omega}$ can be written as

$$
\mathrm{id}_{\mathcal{H}_{\omega}}=Q-\sum_{\alpha= \pm} \sum_{m=1}^{\infty} \frac{\alpha}{m} \chi_{-m}^{\alpha} Q \chi_{m}^{-\alpha}-\sum_{m, n=1}^{\infty} \frac{1}{m n} \chi_{-m}^{+} \chi_{-n}^{-} Q \chi_{n}^{+} \chi_{m}^{-}+R
$$

where $R$ contains only terms with three or more fermion modes on both sides of the projector $Q$. Inserting this into (E.1) gives

$$
\begin{aligned}
& Q V_{A}(a, z)(-1)^{\varepsilon F} V_{B}(b, w) Q \\
& =V_{A}^{0}(a, z)(-1)^{\varepsilon F} V_{B}^{0}(b, w) \\
& \quad-\sum_{\alpha= \pm} \sum_{m=1}^{\infty} \frac{\alpha}{m}\left(\frac{w}{z}\right)^{m}(-1)^{\varepsilon} V_{A}^{0}\left((-1)^{F} \chi_{0}^{\alpha} a, z\right)(-1)^{\varepsilon F} V_{B}^{0}\left(\chi_{0}^{-\alpha} b, w\right) \\
& \quad-\sum_{m, n=1}^{\infty} \frac{1}{m n}\left(\frac{w}{z}\right)^{m+n} V_{A}^{0}\left(\chi_{0}^{+} \chi_{0}^{-} a, z\right)(-1)^{\varepsilon F} V_{B}^{0}\left(\chi_{0}^{+} \chi_{0}^{-} b, w\right) \\
& =V_{A}^{0}(a, z)(-1)^{\varepsilon F} V_{B}^{0}(b, w) \\
& \quad+(-1)^{\varepsilon} \log \frac{z-w}{z} \sum_{\alpha= \pm} \alpha V_{A}^{0}\left((-1)^{F} \chi_{0}^{\alpha} a, z\right)(-1)^{\varepsilon F} V_{B}^{0}\left(\chi_{0}^{-\alpha} b, w\right) \\
& \quad-\left(\log \frac{z-w}{z}\right)^{2} V_{A}^{0}\left(\chi_{0}^{+} \chi_{0}^{-} a, z\right)(-1)^{\varepsilon F} V_{B}^{0}\left(\chi_{0}^{+} \chi_{0}^{-} b, w\right) .
\end{aligned}
$$

The same calculation can be carried out to compute the product of intertwiners in the 
crossed channel. The result is, for $a, b \in \mathcal{H}_{\omega}^{0}$ and $C, D \in L\left(\mathcal{H}_{\omega}^{0}, \mathcal{H}_{\omega}^{0}\right)$,

$$
\begin{aligned}
& Q V_{C}\left((-1)^{\varepsilon F} V_{D}(a, z-w) b, w\right) Q \\
& =V_{C}^{0}\left((-1)^{\varepsilon F} V_{D}^{0}(a, z-w) b, w\right) \\
& \quad+\log \frac{z}{w} \sum_{\alpha= \pm} \alpha V_{C}^{0}\left((-1)^{(\varepsilon+1)(F+1)} V_{D}^{0}\left(\chi_{0}^{-\alpha} a, z-w\right) b, w\right) \chi_{0}^{\alpha} \\
& \quad-\left(\log \frac{z}{w}\right)^{2} V_{C}^{0}\left((-1)^{\varepsilon F} V_{D}^{0}\left(\chi_{0}^{+} \chi_{0}^{-} a, z-w\right) b, w\right) \chi_{0}^{+} \chi_{0}^{-} .
\end{aligned}
$$

\section{E.2 The $\mu \mu \mu \mu$-block}

The next block we are interested in is

$$
F(x)=\mu^{*} \circ \widetilde{U}_{\varphi}(\mu, 1)(-1)^{\varepsilon F} U_{a}(\mu, x) \mu,
$$

where $\varphi \in\left(\mathcal{H}_{\omega}^{0}\right)^{*}$ and $a \in \mathcal{H}_{\omega}^{0}$. We have also set $z=1$ for the first intertwiner since this is the form in which the block is used in the main text.

The block can be computed using the level 2 Virasoro null vector $N=\left(L_{-2}-2 L_{-1}^{2}\right) \mu=0$ in $\mathcal{H}_{\mu}$. Denote by $f(x)=\langle\mu \mid \mu(1) \mu(x) \mu(0)\rangle$ a conformal block with insertions of $\mu$ at $0,1, x$ and $\infty$. The null vector implies that

$$
\langle\mu \mid N(1) \mu(x) \mu(0)\rangle=0 .
$$

Applying the usual contour deformation arguments gives a second order differential equation for $f(x)$, namely

$$
16 x^{2}(x-1)^{2} f^{\prime \prime}(x)+8 x(x-1)(2 x-1) f^{\prime}(x)+f(x)=0 .
$$

The space of solutions is spanned by

$$
\begin{aligned}
& f_{1}(x)=(x(1-x))^{\frac{1}{4}}{ }_{2} F_{1}\left(\frac{1}{2}, \frac{1}{2} ; 1 ; x\right)=x^{\frac{1}{4}}\left(1-\frac{1}{64} x^{2}+\mathcal{O}\left(x^{3}\right)\right) \\
& f_{2}(x)=(x(1-x))^{\frac{1}{4}}{ }_{2} F_{1}\left(\frac{1}{2}, \frac{1}{2} ; 1 ; 1-x\right)=x^{\frac{1}{4}}\left(\frac{4}{\pi} \log 2-\frac{1}{\pi} \log x-\frac{1}{2 \pi} x+\mathcal{O}_{\log }\left(x^{2}\right)\right)
\end{aligned}
$$

i.e. we find that

$$
\langle\mu \mid \mu(1) \mu(x) \mu(0)\rangle=C_{1} f_{1}(x)+C_{2} f_{2}(x)
$$

for some constants $C_{1}, C_{2}$.

The product of intertwiners $F(x)$ given in (E.6) also solves the differential equation (E.8) and therefore has to be of the form (E.10). Matching the leading order of $F(x)$ (obtained by combining (D.11) and (D.14) $)$ against the asymptotics of the solutions in (E.9) fixes the constants $C_{1}$ and $C_{2}$ in (E.10) uniquely, and one finds

$$
\begin{aligned}
& \mu^{*} \circ \widetilde{U}_{\varphi}(\mu, 1)(-1)^{\varepsilon F} U_{a}(\mu, x) \mu \\
& =(x(1-x))^{\frac{1}{4}}\left\{\varphi\left((-1)^{\varepsilon F}\left(1-4 \log 2 \cdot \chi_{0}^{+} \chi_{0}^{-}\right) a\right){ }_{2} F_{1}\left(\frac{1}{2}, \frac{1}{2} ; 1 ; x\right)\right. \\
& \left.\quad+\pi \varphi\left((-1)^{\varepsilon F} \chi_{0}^{+} \chi_{0}^{-} a\right){ }_{2} F_{1}\left(\frac{1}{2}, \frac{1}{2} ; 1 ; 1-x\right)\right\} .
\end{aligned}
$$


It follows that the space of blocks of type $\langle\mu \mid \mu(1) \mu(x) \mu(0)\rangle$ is given by the product of intertwiners $\mu^{*} \circ \widetilde{U}_{\varphi}(\mu, 1)(-1)^{\varepsilon F} U_{a}(\mu, x) \mu$ for all possible choices of $\varphi$ and $a$. This is in accordance with the analysis of the fusion of representations of the triplet algebra [3], which implies that only the representation $\mathcal{H}_{\omega}$ appears in the fusion of $\mathcal{H}_{\mu}$ with itself.

\section{E.3 The $\mu \mu \omega \omega$-block}

Let

$$
g(x)=\langle\mu \mid \mu(1) \omega(x) \omega(0)\rangle
$$

be a conformal block with states $\omega, \omega, \mu$ and $\mu$ inserted at $0,1, x$ and $\infty$, respectively. To obtain the differential equation from inserting the null vector $N=\left(L_{-2}-2 L_{-1}^{2}\right) \mu$ at the point 1 , the following identity is helpful,

$$
\begin{gathered}
\left\langle\mu \mid \mu(1) \psi(x)\left(L_{-1} \xi\right)(0)\right\rangle=\left\langle\mu \mid \mu(1) \psi(x)\left(L_{0} \xi\right)(0)\right\rangle+\left\langle\mu \mid \mu(1)\left(L_{0} \psi\right)(x) \xi(0)\right\rangle \\
+(x-1)\left\langle\mu \mid \mu(1)\left(L_{-1} \psi\right)(x) \xi(0)\right\rangle,
\end{gathered}
$$

where $\psi$ is a Virasoro highest weight state and $\xi$ is arbitrary. With the help of this identity it is also straightforward to determine the conformal blocks obtained by replacing some of the $\omega$ insertions in $g(x)$ by $\Omega$ insertions,

$$
\begin{aligned}
& \langle\mu \mid \mu(1) \Omega(x) \Omega(0)\rangle=C_{1}, \quad\langle\mu \mid \mu(1) \Omega(x) \omega(0)\rangle=C_{2}, \\
& \langle\mu \mid \mu(1) \omega(x) \Omega(0)\rangle=-C_{1} \log (1-x)+C_{3},
\end{aligned}
$$

where $C_{1}, C_{2}, C_{3} \in \mathbb{C}$ are integration constants. Putting everything together, one computes the following differential equation for $g(x)$,

$$
2 x^{2} g^{\prime \prime}(x)-\frac{x(3 x-2)}{1-x} g^{\prime}(x)+\frac{4 x}{1-x} C_{1}+\frac{x(x-2)}{(1-x)^{2}} C_{2}=0 .
$$

It is solved by

$$
g(x)=-C_{1} h(x)^{2}-C_{2} \log (1-x)+C_{4} h(x)+C_{5}, \quad h(x)=\log \left(\frac{1-\sqrt{1-x}}{1+\sqrt{1-x}}\right) .
$$

Here $C_{4}, C_{5} \in \mathbb{C}$ are two more integration constants.

The same considerations which determined the space of function (E.16), to which conformal blocks of the form $\langle\mu \mid \mu(1) \omega(x) \omega(0)\rangle$ belong, can be used to determine the product of intertwiners

$$
G(x)=\mu^{*} \circ \widetilde{U}_{\varphi}(\mu, 1)(-1)^{\varepsilon F} V_{A}(a, x) Q
$$

with $\varphi \in\left(\mathcal{H}_{\omega}^{0}\right)^{*}, A \in L\left(\mathcal{H}_{\omega}^{0}, \mathcal{H}_{\omega}^{0}\right)$ and $a \in \mathcal{H}_{\omega}^{0}$. One uses the null vector $N$ as well as the identity

$$
\mu^{*} \mu V_{A}(a, x) L_{-1}=\mu^{*} \mu V_{A}\left((x-1) L_{-1} a+L_{0} a, x\right)+\mu^{*} \mu V_{A}(a, x) L_{0},
$$


where we abbreviated $\mu^{*} \mu \equiv \mu^{*} \circ \widetilde{U}_{\varphi}(\mu, 1)(-1)^{\varepsilon F}$, to obtain a differential equation for $G(x)$ analogous to (E.15). The solution one finds is

$$
G(x)=-h(x)^{2} \psi L_{0}-\log (1-x) \psi+h(x) \xi+\beta, \quad \psi:=\varphi \circ(-1)^{\varepsilon F} V_{A}^{0}\left(L_{0} a, x\right),
$$

where $h(x)$ is defined as in (E.16), and $\psi \in\left(\mathcal{H}_{\omega}^{0}\right)^{*}$ can be checked to be independent of $x$. The linear forms $\xi, \beta \in\left(\mathcal{H}_{\omega}^{0}\right)^{*}$ can be fixed by matching the leading term in the small $x$ expansion of (E.17) and (E.19), i.e. by solving

$$
\begin{aligned}
& \varphi \circ(-1)^{\varepsilon F} V_{A}^{0}(a, x) \\
& =\beta-2 \log 2 \cdot\left(\xi+2 \log 2 \cdot \psi L_{0}\right)+\log x \cdot\left(\xi+4 \log 2 \cdot \psi L_{0}\right)-(\log x)^{2} \psi L_{0} .
\end{aligned}
$$

This determines $\xi$ and $\beta$ uniquely. Setting $a=\omega$, the product $\mu^{*} \circ \widetilde{U}_{\varphi}(\mu, 1)(-1)^{\varepsilon F} V_{A}(\omega, x) \omega$ solves the differential equation (E.15). Moreover, the constants $C_{1}=\psi\left(L_{0} \omega\right), C_{2}=\psi(\omega)$, $C_{4}=\xi(\omega)$ and $C_{5}=\beta(\omega)$ appearing in (E.16) can be chosen independently by appropriately varying $A$ and $\varphi$, so that all blocks of type $\langle\mu \mid \mu(1) \omega(x) \omega(0)\rangle$ are of the form (E.17). This is again in accordance with the fusion analysis of [3], which implies that only $\mathcal{H}_{\omega}$ appears in the fusion of $\mathcal{H}_{\mu}$ with itself, and in the fusion of $\mathcal{H}_{\omega}$ with itself.

In the crossed channel the four-point block is written in terms of intertwiners as

$$
\tilde{G}(x)=\mu^{*} \circ \widetilde{U}_{\varphi}\left((-1)^{\varepsilon F} \widetilde{U}_{\psi}(\mu, 1-x) b, x\right) Q
$$

for some $\varphi, \psi \in\left(\mathcal{H}_{\omega}^{0}\right)^{*}$ and $b \in \mathcal{H}_{\omega}^{0}$. One first notes that if $b=L_{0} a$ for some $a$, then $\tilde{G}(x)$ does not depend on $x$. We can thus evaluate it in the limit $x \rightarrow 1$ and find

$$
\mu^{*} \circ \widetilde{U}_{\varphi}\left((-1)^{\varepsilon F} \widetilde{U}_{\psi}(\mu, 1-x) L_{0} a, x\right) Q=\left\langle\psi, L_{0} a\right\rangle \varphi
$$

Again, $\tilde{G}(x)$ solves a differential equation similar to (E.15), but this time with $C_{1}$ replaced by $\left\langle\psi, L_{0} a\right\rangle \varphi L_{0}$ and $C_{2}$ replaced by $\left\langle\psi, L_{0} a\right\rangle \varphi$. The solution is

$$
\tilde{G}(x)=-h(x)^{2}\left\langle\psi, L_{0} a\right\rangle \varphi L_{0}-\log (1-x)\left\langle\psi, L_{0} a\right\rangle \varphi+h(x) \xi^{\prime}+\beta^{\prime},
$$

where the integration constants $\xi^{\prime}, \beta^{\prime} \in\left(\mathcal{H}_{\omega}^{0}\right)^{*}$ are fixed by matching the $x \rightarrow 1$ asymptotics of (E.21) and (E.23), i.e. by solving (setting $x=1-\delta$ )

$$
\begin{aligned}
& \langle\psi, a\rangle \varphi-\log \delta \cdot\left\langle\psi, L_{0} a\right\rangle \varphi+2 \sqrt{\delta} \cdot(-1)^{\varepsilon} \sum_{\alpha= \pm} \alpha \psi\left(\chi_{0}^{-\alpha} a\right) \varphi \chi_{0}^{\alpha}+\mathcal{O}_{\log }(\delta) \\
& \quad=\beta^{\prime}-\log \delta \cdot\left\langle\psi, L_{0} a\right\rangle \varphi-2 \sqrt{\delta} \xi^{\prime}+\mathcal{O}_{\log }(\delta)
\end{aligned}
$$

from which one can read off $\xi^{\prime}$ and $\beta^{\prime}$ directly. To work out the left hand side one needs to make use of the identities, which hold for $a \in \mathcal{H}_{\omega}^{0}$,

$$
\mu^{*} \chi_{\frac{1}{2}}^{\alpha} \widetilde{U}(\mu, z) a=i \sqrt{z} \cdot \mu^{*} \widetilde{U}(\mu, z) \chi_{0}^{\alpha} a, \quad \mu^{*} \widetilde{U}\left(\chi_{-\frac{1}{2}}^{\alpha} \mu, z\right) a=\frac{i}{\sqrt{z}} \cdot \mu^{*} \widetilde{U}(\mu, z) \chi_{0}^{\alpha} a
$$

which in turn are obtained by contour deformation arguments. 


\section{E.4 Bosonic intertwiners and blocks}

To obtain the four point blocks for the various representations of the triplet algebra, one has to project the fermionic representations to subspaces of fixed fermion number. Let

$$
P^{\eta}=\frac{1}{2}\left(1+\eta(-1)^{F}\right)
$$

Then for example

$$
u \mapsto P^{+} V_{A}(u, z) P^{+}
$$

with $u$ in $\mathcal{R}_{0} \subset \mathcal{H}_{\omega}$ and $V_{A}(\cdot, z)$ as defined in section D.1 is an intertwiner from $\mathcal{R}_{0} \times \mathcal{R}_{0}$ to $\mathcal{R}_{0}$, i.e. a linear map from $\mathcal{R}_{0}$ to $L\left(\mathcal{R}_{0}, \mathcal{R}_{0}\right) \llbracket z^{ \pm} 1, \log z \rrbracket$.

Accordingly, for example the space of four-point blocks with insertions of $\mu$ at $0,1, x$ and $\infty$ and with $\mathcal{R}_{1}$ running in the intermediate channel is given by

$$
\left\langle\mu^{*}, \widetilde{U}_{\varphi}(\mu, 1) P^{-} U_{a}(\mu, x) \mu\right\rangle,
$$

with $\varphi$ and $a$ taking values in $\left(\mathcal{H}_{\omega}^{0}\right)^{*}$ and $\mathcal{H}_{\omega}^{0}$, respectively. Note that distinct choices of $\varphi$ and $a$ do not necessarily result in different 4 -point blocks.

\section{F Associativity of the boundary fields}

\section{F.1 Associative, unital algebras on $\mathcal{R}_{0}$}

The consistency of the OPE of boundary fields on the $(\mathrm{N}, \pm)$ boundary with factorisation (or crossing) can also be formulated on the level of intertwiners. For $u \in \mathcal{R}_{0}$ denote by

$$
\Lambda_{M}(u, x)=P^{+} V_{M}(u, x) P^{+}
$$

the intertwiner of $\mathcal{R}_{0}$ representations as in equation (E.27), with $P^{+}$the projector (E.26) on the subspace $\mathcal{R}_{0} \subset \mathcal{H}_{\omega}$, and $M \in L\left(\mathcal{H}_{\omega}^{0}, \mathcal{H}_{\omega}^{0}\right)$ (see the end of section D.1). The OPE of two boundary fields $\psi, \psi^{\prime} \in \mathcal{H}^{\text {bnd }} \equiv \mathcal{R}_{0}$ can be written as

$$
\psi(x) \psi^{\prime}(0)=\Lambda_{M}(\psi, x) \psi^{\prime} .
$$

Factorisation of the boundary OPE can now be formulated as an associativity condition for the intertwiners $V_{M}$, i.e. one has to find a $M \in L\left(\mathcal{H}_{\omega}^{0}, \mathcal{H}_{\omega}^{0}\right)$ such that

$$
\Lambda_{M}(\psi, x) \Lambda_{M}\left(\psi^{\prime}, y\right)=\Lambda_{M}\left(\Lambda_{M}(\psi, x-y) \psi^{\prime}, y\right)
$$

for all $\psi, \psi^{\prime} \in \mathcal{R}_{0}$. The condition that $\Omega(x)$ should be the identity field in turn reads

$$
\Lambda_{M}(\Omega, x)=\mathrm{id}_{\mathcal{R}_{0}}
$$

Phrased in the language of the representation category of the triplet algebra, conditions (F.3) and (F.4 amount to endowing the object $\mathcal{R}_{0}$ with the structure of a unital, associative algebra. Let us denote this algebra by $A_{M}$. The algebra $A_{M}$ is a logarithmic analog of the 
special symmetric Frobenius algebras used in [57] to describe local conformal field theories, or of the open string vertex algebras of [65].

The solutions to (F.3) and (F.4 can be written out explicitly. To find them one uses the conformal blocks given in (E.4) and (E.5). This results in a number of linear and quadratic relations for $M$ (which one can conveniently keep track of with a computer algebra program). Let us choose the basis

$$
v_{1}=\omega, \quad v_{2}=\chi_{0}^{+} \omega, \quad v_{3}=\chi_{0}^{-} \omega, \quad v_{4}=\chi_{0}^{+} \chi_{0}^{-} \omega
$$

of $\mathcal{H}_{\omega}$, so that we can express $M$ as a $4 \times 4$-matrix $\left(m_{i j}\right)$. Because of the projectors, the intertwiner $\Lambda_{M}(\cdot, z)$ will only depend on the 8 entries

$$
M=\left(\begin{array}{cccc}
m_{11} & * & * & m_{14} \\
* & m_{22} & m_{23} & * \\
* & m_{32} & m_{33} & * \\
m_{41} & * & * & m_{44}
\end{array}\right)
$$

of $M$. Requiring ( $(\underline{F .3})$ and $(\underline{F .4})$ to hold is now equivalent to (upon setting the irrelevant entries of $M$ to zero)

$$
M=\left(\begin{array}{cccc}
m_{11} & 0 & 0 & -1 \\
0 & m_{22} & m_{23} & 0 \\
0 & m_{32} & m_{11}-m_{22} & 0 \\
m_{22}\left(m_{11}-m_{22}\right)-m_{23} m_{32} & 0 & 0 & 0
\end{array}\right) .
$$

In particular, there are four free parameters $m_{11}, m_{22}, m_{23}, m_{32} \in \mathbb{C}$. Some of these solutions will be isomorphic in the sense that they can be related via

$$
\Lambda_{M^{\prime}}(u, z)=f^{-1} \Lambda_{M}(f u, z) f
$$

where $f: \mathcal{R}_{0} \rightarrow \mathcal{R}_{0}$ is an isomorphism of representations. Since $f$ is determined uniquely by $f(\omega)$, the space of such isomorphisms is two-dimensional. In addition, $f$ has to preserve the unit of the algebra, $f(\Omega)=\Omega$, which leaves a one-dimensional space. The freedom (F.8) can thus be used to remove one of the parameters, for example one can always achieve $m_{11}=0$. The leading term in the OPE of $\omega$ with itself then reads

$$
\omega(x) \omega=\Lambda_{M}(\omega, x) \omega=-2 \log x \cdot \omega+\left(\left(m_{22}\right)^{2}+m_{23} m_{32}-(\log x)^{2}\right) \Omega+\mathcal{O}_{\log }[h=1, x],
$$

which is indeed of the form (4.5).

For non-logarithmic rational conformal field theories one finds [57, 59] that every algebra of boundary fields with certain extra properties (it has to be special symmetric Frobenius) gives rise to a consistent local conformal field theory. The construction of [57, 59] does not apply in the present case, but it would nonetheless be interesting to investigate if also other solutions of the family (F.7) describe the algebra of boundary fields for a consistent conformal field theory (for which the chiral symmetry contains the triplet algebra). 


\section{F.2 $\mathcal{V}_{-\frac{1}{8}}$ as a representation of the boundary algebra}

The analysis of the cylinder partition functions in section 3.3 showed that the space boundary changing fields form $(\mathrm{D}, \eta)$ to $(\mathrm{N}, \eta)$ is isomorphic to $\mathcal{V}_{-\frac{1}{8}}$. For $u \in \mathcal{V}_{-\frac{1}{8}}$ and $\rho \in\left(\mathcal{H}_{\omega}^{0}\right)^{*}$ denote by

$$
R_{\rho}(u, z)=P^{+} \widetilde{U}_{\rho}(u, z) P^{+}
$$

the intertwiner of type $\mathcal{V}_{-\frac{1}{8}} \times \mathcal{R}_{0} \rightarrow \mathcal{V}_{-\frac{1}{8}}$ obtained by projecting $\widetilde{U}_{\rho}(\cdot, z)$. The OPE of a boundary changing field $\xi(x)$ with a boundary field $\psi(0)$ on the $(\mathrm{N}, \eta)$ boundary can then be written as

$$
\xi(x) \psi(0)=R_{\rho}(\xi, x) \psi,
$$

and the factorisation condition takes the form

$$
R_{\rho}\left(R_{\rho}(\xi, x-y) \psi, y\right)=R_{\rho}(\xi, x) \Lambda_{M}(\psi, y) .
$$

Demanding that $\Omega$ acts as the identity can be formulated as

$$
\lim _{x \rightarrow 0} R_{\rho}(\xi, x) \Omega=\xi .
$$

These two equations, which have to hold for all $\xi \in \mathcal{V}_{-\frac{1}{8}}$ and $\psi \in \mathcal{R}_{0}$ result in constraints on $\rho$ and $M$. Using the two blocks $(\mathbb{E . 1 9})$ and $(\mathbb{E . 2 3})$ one finds the constraints to be, in terms of the dual of the basis (F.5), and in terms of the components $m_{11}, \ldots$ of the linear map $M$ in (F.7),

$$
\rho=\left(\frac{1}{2} m_{11}-2 \log 2\right) v_{1}^{*}-v_{4}^{*}, \quad\left(m_{11}-2 m_{22}\right)^{2}=-4 m_{23} m_{32} .
$$

In particular, it thus follows that the OPE of $\xi$ and $\psi$ is uniquely determined by the OPE on $(\mathrm{N}, \pm)$. Furthermore, not all values for $M$ are consistent with the requirement that $\mathcal{V}_{-\frac{1}{8}}$ is a space of boundary changing fields. Note also that (F.14) together with $m_{11}=0$ fixes the leading term in the OPE (F.9) completely, and one obtains precisely (2.23).

Nonetheless, the analysis so far does not fully determine the OPE of $\omega$ with itself beyond leading order; even after fixing the freedom to redefine $\omega$ by setting $m_{11}=0$, we still have not determined the values of $m_{23}$ and $m_{32}$. While it seems likely that these two constants can be fixed by other factorisation considerations, for the present paper we content ourselves with knowing the leading order.

In the language of algebras and representations, the conditions (F.12) and (F.13) define a (right-)module of the unital algebra $A_{M}$ defined in section F.1] The conditions (F.14) then mean that only for specific choices of $M$ is it possible to endow the object $\mathcal{V}_{-\frac{1}{8}}$ with the structure of an $A_{M}$ module. However, if it is possible, the module structure is unique.

\section{References}

[1] V. Gurarie, Logarithmic operators in conformal field theory, Nucl. Phys. B 410 (1993) 535 [hep-th/9303160].

[2] L. Rozansky and H. Saleur, Quantum field theory for the multi-variable AlexanderConway polynomial, Nucl. Phys. B 376 (1992) 461 [hep-th/9203069]. 
[3] M.R. Gaberdiel and H.G. Kausch, A rational logarithmic conformal field theory, Phys. Lett. B 386 (1996) 131 [hep-th/9606050].

[4] M.A.I. Flohr, Bits and pieces in logarithmic conformal field theory, Int. J. Mod. Phys. A 18 (2003) 4497 [hep-th/0111228].

[5] M.R. Gaberdiel, An algebraic approach to logarithmic conformal field theory, Int. J. Mod. Phys. A 18 (2003) 4593 [hep-th/0111260].

[6] S. Kawai, Logarithmic conformal field theory with boundary, Int. J. Mod. Phys. A 18 (2003) 4655 [hep-th/0204169].

[7] H. Saleur, Polymers and percolation in two dimensions and twisted $N=2$ supersymmetry, Nucl. Phys. B 382 (1992) 486 [hep-th/9111007].

[8] M.A.I. Flohr, On modular invariant partition functions of conformal field theories with logarithmic operators, Int. Journ. Mod. Phys. A 11 (1996) 4147 [hep-th/9509166].

[9] H.G. Kausch, Curiosities at $c=-2$, preprint DAMTP 95-52, hep-th/9510149.

[10] G.M.T. Watts, A crossing probability for critical percolation in two dimensions, J. Phys. A 29 (1996) L363 [cond-mat/9603167].

[11] M.A.I. Flohr and A. Müller-Lohmann, Conformal field theory properties of two-dimensional percolation, J. Stat. Mech. (2005) P12006 [hep-th/0507211].

[12] J.-S. Caux, I.I. Kogan and A.M. Tsvelik, Logarithmic operators and hidden continuous symmetry in critical disordered models, Nucl. Phys. B 466 (1996) 444 [hep-th/ 9511134].

[13] Z. Maassarani and D. Serban, Non-unitary conformal field theory and logarithmic operators for disordered systems, Nucl. Phys. B 489 (1997) 603 [hep-th/9605062].

[14] J.-S. Caux, N. Taniguchi and A.M. Tsvelik, Disordered Dirac fermions: multifractality, termination and logarithmic conformal field theories, Nucl. Phys. B 525 (1998) 671 [cond-mat/9801055].

[15] V. Gurarie and A.W.W. Ludwig, Conformal algebras of 2D disordered systems, J. Phys. A 35 (2002) L377 [cond-mat/9911392].

[16] P. Ruelle, $A c=-2$ boundary changing operator for the abelian sandpile model, Phys. Lett. B 539 (2002) 172 [hep-th/0203105].

[17] J. de Gier, B. Nienhuis, P.A. Pearce and V. Rittenberg, The raise and peel model of a fluctuating interface, J. Statist. Phys. 114 (2004) 1 [cond-mat/0301430].

[18] G. Piroux and P. Ruelle, Pre-logarithmic and logarithmic fields in sandpile model, J. Stat. Mech. (2004) P10005 [hep-th/0407143]. 
[19] S. Moghimi-Araghi, M.A. Rajabpour and S. Rouhani, Abelian sandpile model: a conformal field theory point of view, Nucl. Phys. B 718 (2005) 362 [cond-mat/0410434].

[20] M. Jeng, G. Piroux and P. Ruelle, Height variables in the Abelian sandpile model: scaling fields and correlations, cond-mat/0609284.

[21] P.A. Pearce, J. Rasmussen and J.-B. Zuber, Logarithmic minimal models, hep-th/ 0607232.

[22] I.I. Kogan and N.E. Mavromatos, World-sheet logarithmic operators and target space symmetries in string theory, Phys. Lett. B 375 (1996) 111 [hep-th/9512210].

[23] V. Periwal and O. Tafjord, D-brane recoil, Phys. Rev. D 54 (1996) 3690 [hep-th/ 9603156].

[24] I.I. Kogan, N.E. Mavromatos and J.F. Wheater, D-Brane recoil and logarithmic operators, Phys. Lett. B 387 (1996) 483 [hep-th/9606102].

[25] N. Lambert, H. Liu and J. Maldacena, Closed strings from decaying D-branes, hep-th/ 0303139.

[26] I. Bakas and K. Sfetsos, PP-waves and logarithmic conformal field theories, Nucl. Phys. B 639 (2002) 223 [hep-th/0205006].

[27] A. Milas, Weak modules and logarithmic intertwining operators for vertex operator algebras, Contemp. Math. 297 (2002) 201 [math.qa/0101167].

[28] M. Miyamoto, Modular invariance of vertex operator algebras satisfying $\mathrm{C}_{2}$-cofiniteness, math.QA/0209101.

[29] Y.Z. Huang, J. Lepowsky and L. Zhang, A logarithmic generalization of tensor product theory for modules for a vertex operator algebra, Int. J. of Math. 17 (2006) 975 [math.qa/0311235].

[30] V. Gurarie and A.W.W. Ludwig, Conformal field theory at central charge $c=0$ and two-dimensional critical systems with quenched disorder, in: Shifman, M. et al (eds.): 'From fields to strings' vol. 2, 1384, World Scientific (2005) [hep-th/0409105].

[31] V. Schomerus and H. Saleur, The GL(1|1) WZW model: From supergeometry to logarithmic CFT, Nucl. Phys. B 734 (2006) 221 [hep-th/0510032].

[32] M.R. Gaberdiel, Fusion rules and logarithmic representations of a WZW model at fractional level, Nucl. Phys. B 618 (2001) 407 [hep-th/0105046].

[33] J. Fjelstad, J. Fuchs, S. Hwang, A.M. Semikhatov and I.Y. Tipunin, Logarithmic conformal field theories via logarithmic deformations, Nucl. Phys. B 633 (2002) 379 [hep-th/0201091]. 
[34] F. Lesage, P. Mathieu, J. Rasmussen and H. Saleur, The $\widehat{s u(2)}-1 / 2$ WZW model and the beta-gamma system, Nucl. Phys. B 647 (2002) 363 [hep-th/0207201].

[35] J. Rasmussen, Affine Jordan cells, logarithmic correlators, and Hamiltonian reduction, Nucl. Phys. B 736 (2006) 225 [hep-th/0508179].

[36] N.S. Izmailian, V.B. Priezzhev, P. Ruelle and C.-K. Hu, Logarithmic conformal field theory and boundary effects in the dimer model, Phys. Rev. Lett. 95 (2005) 260602 [cond-mat/0512703].

[37] S. Moghimi-Araghi and S. Rouhani, Logarithmic conformal field theories near a boundary, Lett. Math. Phys. 53 (2000) 49 [hep-th/0002142].

[38] I.I. Kogan and J.F. Wheater, Boundary logarithmic conformal field theory, Phys. Lett. B 486 (2000) 353 [hep-th/0003184].

[39] Y. Ishimoto, Boundary states in boundary logarithmic CFT, Nucl. Phys. B 619 (2001) 415 [hep-th/0103064].

[40] S. Kawai and J.F. Wheater, Modular transformation and boundary states in logarithmic conformal field theory, Phys. Lett. B 508 (2001) 203 [hep-th/0103197].

[41] A. Bredthauer and M.A.I. Flohr, Boundary states in $c=-2$ logarithmic conformal field theory, Nucl. Phys. B 639 (2002) 450 [hep-th/0204154].

[42] A. Bredthauer, Boundary states and symplectic fermions, Phys. Lett. B 551 (2003) 378 [hep-th/0207181].

[43] M.R. Gaberdiel and H.G. Kausch, A local logarithmic conformal field theory, Nucl. Phys. B 538 (1999) 631 [hep-th/9807091].

[44] H.G. Kausch, Extended conformal algebras generated by a multiplet of primary fields, Phys. Lett. B 259 (1991) 448.

[45] H.G. Kausch, Symplectic fermions, Nucl. Phys. B 583 (2000) 513 [hep-th/0003029].

[46] M.R. Gaberdiel and H.G. Kausch, Indecomposable fusion products, Nucl. Phys. B 477 (1996) 293 [hep-th/9604026].

[47] T. Abe, $A \mathbb{Z}_{2}$-orbifold model of the symplectic fermionic vertex operator subalgebra, math.QA/0503472.

[48] N. Carqueville and M.A.I. Flohr, Nonmeromorphic operator product expansion and $C_{2^{-}}$ cofiniteness for a family of W-algebras, J. Phys. A 39 (2006) 951 [math-ph/0508015].

[49] J. Fuchs, S. Hwang, A.M. Semikhatov and I.Y. Tipunin, Nonsemisimple fusion algebras and the Verlinde formula, Commun. Math. Phys. 247 (2004) 713 [hep-th/0306274]. 
[50] B.L. Feigin, A.M. Gainutdinov, A.M. Semikhatov and I.Y. Tipunin, Kazhdan-Lusztig correspondence for the representation category of the triplet $W$-algebra in logarithmic conformal field theory, math.qa/0512621.

[51] M.A.I. Flohr and M.R. Gaberdiel, Logarithmic torus amplitudes, J. Phys. A 39 (2006) 1955 [hep-th/0509075].

[52] M.A.I. Flohr, On Fusion Rules in Logarithmic Conformal Field Theories, Int. J. Mod. Phys. A 12 (1997) 1943 [hep-th/9605151].

[53] B.L. Feigin, A.M. Gainutdinov, A.M. Semikhatov and I.Y. Tipunin, Modular group representations and fusion in logarithmic conformal field theories and in the quantum group center, Commun. Math. Phys. 265 (2006) 47 [hep-th/0504093].

[54] J. Fuchs, On non-semisimple fusion rules and tensor categories, hep-th/0602051.

[55] J.L. Cardy, Boundary conditions, fusion rules and the Verlinde formula, Nucl. Phys. B 324 (1989) 581.

[56] J.L. Cardy and D.C. Lewellen, Bulk and boundary operators in conformal field theory, Phys. Lett. B 259 (1991) 274.

[57] J. Fuchs, I. Runkel and C. Schweigert, TFT construction of RCFT correlators. I: Partition functions, Nucl. Phys. B 646 (2002) 353 [hep-th/0204148].

[58] J. Fuchs, I. Runkel and C. Schweigert, TFT construction of RCFT correlators. IV: Structure constants and correlation functions, Nucl. Phys. B 715 (2005) 539 [hep-th/0412290].

[59] J. Fjelstad, J. Fuchs, I. Runkel and C. Schweigert, TFT construction of RCFT correlators. V: Proof of modular invariance and factorisation, Theo. and Appl. of Cat. 16 (2006) 342 [hep-th/0503194].

[60] M.R. Gaberdiel, A general transformation formula for conformal fields, Phys. Lett. B 325 (1994) 366 [hep-th/9401166].

[61] E. Frenkel and D. Ben-Zvi, Vertex algebras and algebraic curves, Math. surveys and monographs, vol 88, AMS (2004) [2nd ed], www.math.berkeley.edu/ frenkel/BOOK.

[62] V.G. Kac and W. Wang, Vertex operator superalgebras and their representations, Contemp. Math. 175 (1994) 161 [hep-th/9312065].

[63] C. Dong, H. Li and G. Mason, Twisted representations of vertex operator algebras, Intern. Math. Res. Notices 8 (1998) 389 [q-alg/9509005].

[64] M.R. Gaberdiel, Fusion of twisted representations, Int. J. Mod. Phys. A 12 (1997) 5183 [hep-th/9607036].

[65] Y.-Z. Huang and L. Kong, Open-string vertex algebras, tensor categories and operads, Commun. Math. Phys. 250 (2004) 433 [math.qa/0308248]. 